\title{
CLASSES OF MAXIMUM NUMBERS AND MINIMUM NUMBERS THAT ARE ASSOCIATED WITH CERTAIN SYMMETRIC EQUATIONS IN $n$ RECIPROCALS*
}

\author{
BY \\ II. A. SIMMONS
}

1. Introduction. Recently we presented a solution in positive integers of the equation $\dagger$

$$
\Sigma\left(1 /\left(x_{1} x_{2} \cdots x_{r}\right)\right)=b / a, a \equiv[(c+1) b-1],
$$

in which $b, c$ are positive integers and $\Sigma\left(1 /\left(x_{1} x_{2} \cdots x_{r}\right)\right)$ is the $r$ th elementary symmetric function of the $n, n>r$, reciprocals $1 / x_{1}, 1 / x_{2}, \cdots, 1 / x_{n}$; and we proposed the problems of finding the maximum number and the maximum sum and the maximum product of the numbers that can appear in any solution in positive integers of (1).

The purposes of the present paper are as follows: to obtain a result that includes as a special case a solution of the problem concerning the maximum number just mentioned; to identify relative to (1) a class of maximum numbers that includes the maximum sum and the maximum product (but not the maximum number) just referred to, and to state without proof results that we have obtained concerning classes of maximum numbers relative to certain elementary symmetric equations that include (1); to identify relative to a very general symmetric (not necessarily elementary symmetric) equation in $n, n>1$, reciprocals a class of minimum numbers; and to give applications of some of these results.

A reader who desires only a statement of our main results and the applications that we give of them should refer to $\$ \$ 7$ and 12 for our definitions of $E$-solution and $\Sigma_{i, j}(x)$, respectively, and then read the two theorems in $\$ 12$, the two in $\$ 23$, and the applications in $\$ \$ 24$ to 27 inclusive. Theorem $2, \S 12$, contains our first generalization of the known results concerning Kellogg's Diophantine problem $\ddagger$ and extensions of it. Theorem $3, \S 12$, defines the class of maximum numbers that we associate with (1). Our last application, $\$ 27$, contains for a perfect number with exactly $n$ divisors less than itself an apparently new upper bound in which number theorists may be interested.

* Presented to the Society, April 4, 1931, 1)ecember 28, 1931, and April 9, 1932; received by the editors April 8, 1932.

$\dagger$ Cf. American Mathematical Monthly, 1930, p. 141.

‡ C. . O. 1). Kellogg, American Mathematical Monthly, 1921, p. 300; 1). R. Curtiss, American Mathematical Monthly, 1922, pp. 386-387; and Tanzô Takenouchi, Proceedings of the PhysicoMathematical Society of Japan, (3), vol. 3, No. 6, pp. 78-92. 
Irrational, as well as rational, numbers are included both among the maximum numbers and the minimum numbers that we identify, so that our results are not of a purely Diophantine character.

The discussion from $\$ 2$ to the end of this paper is divided into five parts, as follows: Part 1, a class of minimum numbers, $\$ \S 2$ to 5 (inclusive); Part 2, a general approach to our theory of maximum numbers, $\$ \S 6$ to 11; Part 3, the individual maximum number and the class of maximum numbers that we associate with equation (1), $\$ 12$ to 21 ; Part 4, further possibilities of the procedure of Part 3, $\$ \$ 22$ to 23; Part 5, applications (to series, theory of equations, a problem in physics, and perfect numbers), $\$ \$ 24$ to 27 .

We present our theory of minimum numbers first because we are able to give it briefly and at the same time prepare the reader, to some extent, for the more lengthy discussion of maximum numbers.

\section{Part 1. A CLASS OF Minimum Numbers}

2. Statement of Theorem 1. Let $Q\left(1 / x_{1}, 1 / x_{2}, \cdots, 1 / x_{n}\right) \equiv Q(1 / x)$ be any polynomial that is symmetric in the $n, n>1$, reciprocals $1 / x_{1}, 1 / x_{2}, \cdots$, $1 / x_{n}$, contains one or more positive coefficients and no negative coefficient, and has no constant term. We wish to identify a class of minimum numbers relative to the equation

$$
Q(1 / x)=c,
$$

where $c$ is any positive constant and the $x_{p}(p=1, \cdots, n)$ are restricted to positive values.

If in (2) we set each of the $x_{p}$ equal to $X$, the resulting equation will have, according to a well known theorem (for algebraic equations) and the definition of $Q(1 / x)$, exactly one positive root, say $X=M$. Thus one positive solution* of (2) is the symmetrical solution $x=W$, where

$$
W_{p}=M \quad(p=1,2, \cdots, n) .
$$

Let $P\left(x_{1}, x_{2}, \cdots, x_{n}\right) \equiv P(x)$ be any polynomial which is symmetric in the $n$ variables that appear in $Q(1 / x)$ and contains one or more positive coefficients and no negative coefficient, and is not identically equal to a constant.

The case in which $Q(1 / x)$ and $P(x)$ are polynomials in $\left(x_{1} x_{2} \cdots x_{n}\right)^{-1}$ and $x_{1} x_{2} \cdots x_{n}, \dagger$ respectively, will be referred to as the special case.

The result which we desire to prove is expressed in the following theorem.

\footnotetext{
* Positive solution means solution in positive numbers.

$\dagger$ That is, in compact language, where $P(x)[Q(1 / x)]$ does not contain any one of its variables except in some positive integral power of $x_{1} x_{2} \cdots x_{n}\left[\left(x_{1} x_{2} \cdots x_{n}\right)^{-1}\right]$.
} 
THEOREM 1. If $x \neq W$ is a positive solution of $(2),{ }^{*} P(x)>P(W)$ except in the special case, in which $P(x)=P(W)$.

In $\$ 3$ we indicate convenient ways for our purposes of expressing $Q(1 / x)$ and $P(x)$ in terms of two of their variables; in $\$ 4$, we exhibit a useful transformation, and we establish a lemma that is to be employed in the proof of Theorem 1 ; in $\$ 5$, we prove Theorem 1 .

3. Expressions for $Q(1 / x)$ and $P(x)$. If $x_{i}$ and $x_{i}$ are any two distinct variables of the set $x$ of $(2)$, then $Q(1 / x)[P(x)]$ can be expressed in exactly one way apart from arrangements of terms as a polynomial in $\left(x_{i} x_{j}\right)^{-1}$ and $\left(x_{i}^{-p}+x_{j}^{-p}\right), p=1,2, \cdots\left[x_{i} x_{j}\right.$ and $\left.\left(x_{i}^{t}+x_{j}^{t}\right), t=1,2, \cdots\right]$; the coefficients being positive and independent of $x_{i}$ and $x_{j}$. Suppose that

$$
\begin{aligned}
Q(1 / x) & \equiv \sum_{(p)} A_{p}\left(x_{i}^{-p}+x_{j}^{-p}\right)+\sum_{(q s)} B_{q s}\left(x_{i} x_{j}\right)^{-q}\left(x_{i}^{-s}+x_{j}^{-s}\right) ; \\
P(x) & \equiv \sum_{(t)} C_{t}\left(x_{i}{ }^{t}+x_{j}{ }^{t}\right)+\sum_{(u v)} D_{u v}\left(x_{i} x_{j}\right)^{u}\left(x_{i}^{v}+x_{j}^{v}\right),
\end{aligned}
$$

where for a given polynomial $Q(1 / x)[P(x)]$ each of $p, q, s[t, u, v]$ ranges over a finite number of non-negative integral values and $q[u]$, let us say, does not assume the value zero.

The use that we make of (4) and (5) in our proof of Theorem 1 will be apparent from the lemma of $\$ 4$.

4. A transformation and a fundamental lemma. We first define the transformation that we use in proving Theorem 1. Since $x$ (of Theorem 1) is different from $W$, there exists in $x$ at least one number $<M$ (cf. (3)) and at least one $>M$. Suppose that $i$ and $j$ are positive integers, each $\leqq n$, such that $x_{i}<M$ and $x_{j}>M$; and apply to $x$ the transformation

$$
x_{p}^{\prime}=x_{p} \quad(p \neq i, j), x_{i}^{\prime}=\left(x_{i}+\alpha\right) \leqq M, x_{j}^{\prime}=\left(x_{j}-\beta\right) \geqq M,
$$

where $\alpha$ and $\beta$ are positive numbers so chosen that the set $x^{\prime}$ satisfies (2). $\dagger$ That $P(x)>P\left(x^{\prime}\right)$ except in the special case is a consequence of equation (5) and the following lemma.

LEMMA 1. With $i$ and $j$ equal to distinct positive integers, each $\leqq n$, if $x_{i}, x_{j}$, $\alpha, \beta$ are positive numbers such that $\left(x_{i}+\alpha\right) \leqq\left(x_{j}-\beta\right) ;$ if $p,(q, s), A_{p}, B_{q s}$ have the meanings here that they have in (4); and if

* There exist infinitely many positive solutions of (2); for $W$ is one such solution, and since the roots of a rational integral algebraic equation are continuous functions of its coefficients, equation (2) has infinitely many positive solutions that differ only slightly from $W$.

$\dagger$ That $\alpha, \beta$ can be so chosen is evident from a fact that was used in the above footnote. 


$$
\begin{aligned}
& \sum_{(p)} A_{p}\left[\left(x_{i}+\alpha\right)^{-p}+\left(x_{j}-\beta\right)^{-p}\right] \\
+ & \sum_{(q s)} B_{q s}\left(x_{i}+\alpha\right)^{-q}\left(x_{j}-\beta\right)^{-q}\left[\left(x_{i}+\alpha\right)^{-s}+\left(x_{j}-\beta\right)^{-s}\right]=Q(1 / x),,^{*}
\end{aligned}
$$

then

$$
x_{i} x_{j} \geqq\left(x_{i}+\alpha\right)\left(x_{j}-\beta\right) ;\left(x_{i}^{h}+x_{j}^{h}\right)>\left[\left(x_{i}+\alpha\right)^{h}+\left(x_{j}-\beta\right)^{h}\right],
$$

where $h$ is a positive integer. Furthermore the equality sign holds in $\left(8_{1}\right)$ if, and only if, $Q(1 / x)$ is a polynomial in $\left(x_{1} x_{2} \cdots x_{n}\right)^{-1}[$ and $Q(1 / x)$ is otherwise as it was defined in \$2].

If we can prove that $\beta>\alpha,\left(8_{2}\right)$ will follow, as can be shown by considering its equivalent

$$
\left[x_{j}^{h}-\left(x_{j}-\beta\right)^{h}\right]>\left[\left(x_{i}+\alpha\right)^{h}-x_{i}^{h}\right]
$$

which readily reduces to

$$
\begin{aligned}
\beta\left[x_{i}^{h-1}+x_{i}^{h-2}\left(x_{i}-\beta\right)+\cdots\right. & \left.+\left(x_{j}-\beta\right)^{h-1}\right] \\
& >\alpha\left[x_{i}^{h-1}+x_{i}^{h-2}\left(x_{i}+\alpha\right)+\cdots+\left(x_{i}+\alpha\right)^{h-1}\right],
\end{aligned}
$$

and observing that, with $\beta>\alpha$ and $\left(x_{i}+\alpha\right) \leqq\left(x_{j}-\beta\right)$,

$$
\beta x_{j}^{k}\left(x_{j}-\beta\right)^{h-1-k}>\alpha x_{i}^{k}\left(x_{i}+\alpha\right)^{h-1-k}(k=0,1, \cdots, h-1) .
$$

Consequently, to prove Lemma 1 , it suffices to show that the following statements are true: (A) $\beta>\alpha$; (B) if $Q(1 / x)$ is a polynomial in $\left(x_{1} x_{2} \cdots x_{n}\right)^{-1}$, the equality sign holds in $\left(8_{1}\right)$; (C) if $Q(1 / x)$ is not such a polynomial, the inequality sign holds in $\left(8_{1}\right)$.

(A) We first prove that $\beta \neq \alpha$. Suppose $\beta=\alpha$. We shall show that this assumption leads to a contradiction of (7). The inequality $x_{i} x_{j}<\left(x_{i}+\alpha\right)\left(x_{j}-\beta\right)$ follows from the hypotheses $\left(x_{i}+\alpha\right) \leqq\left(x_{j}-\beta\right)$ and $\beta=\alpha>0$. Consequently, with $m$ equal to any positive integer,

$$
\left(x_{i}+\alpha\right)^{-m}\left(x_{j}-\alpha\right)^{-m}<\left(x_{i} x_{j}\right)^{-m} .
$$

From our hypotheses, it is easy to prove by procedure that was used in the last paragraph above that

$$
\left[\left(x_{i}+\alpha\right)^{m}+\left(x_{j}-\alpha\right)^{m}\right] \leqq\left(x_{i}^{m}+x_{j}^{m}\right) .
$$

Now (9) and (10) imply that

$$
\left[\left(x_{i}+\alpha\right)^{-m}+\left(x_{j}-\alpha\right)^{-m}\right]<\left(x_{i}^{-m}+x_{j}^{-m}\right) ;
$$

and with $\beta=\alpha,(7),(9),(11)$ involve a contradiction. Hence $\beta \neq \alpha$.

That $\beta>\alpha$ can be proved as follows. Use of (9) and (11) in (7) shows, as

${ }^{*}$ Cf. (4). 
was just observed, that if $\beta=\alpha$ the left member of (7) is less than its right. Further, if $\beta$ is decreased from one positive value to another (the other quantities in (7) remaining fixed) the left member of (7) is decreased. Hence it is evident that if the left member of (7) is as large as its right, then $\beta>\alpha$.

(B) Let $\lambda \equiv\left(x_{i}+\alpha\right)\left(x_{j}-\beta\right)$ and $\mu \equiv x_{i} x_{j}$. In the present case, then, (7) reduces to

$$
\sum_{(q)} B_{q 0}\left(\lambda^{-q}-\mu^{-q}\right)=0 .
$$

By hypothesis $\lambda>0, \mu>0, B_{q 0} \geqq 0$ for every value that $q$ assumes in (12), and $B_{q 0}>0$ for at least one such value of $q$, which is necessarily a positive integer ( $q \neq 0$, cf. §3). Hence (12) has exactly one real solution for $\lambda$, namely $\lambda=\mu$. Therefore the equality sign holds in $\left(8_{1}\right)$.

(C) We again make an indirect proof in which (7) is contradicted. Suppose that $x_{i} x_{j} \leqq\left(x_{i}+\alpha\right)\left(x_{j}-\beta\right)$, so that with $m$ equal to any positive integer,

$$
\left(x_{i}+\alpha\right)^{-m}\left(x_{j}-\beta\right)^{-m} \leqq\left(x_{i} x_{j}\right)^{-m} .
$$

From (13) and the fact that under present hypotheses (one of which is $\beta>\alpha$ )

$$
\left[\left(x_{i}+\alpha\right)^{m}+\left(x_{j}-\beta\right)^{m}\right]<\left(x_{i}^{m}+x_{j}^{m}\right)
$$

(cf. (10)), it follows that

$$
\left[\left(x_{i}+\alpha\right)^{-m}+\left(x_{j}-\beta\right)^{-m}\right]<\left(x_{i}^{-m}+x_{j}^{-m}\right) .
$$

In the present case, either $A_{p}>0$ for some positive integral value of $p$ in (7) or $B_{q 8}>0$ for some pair of positive integral values $q, s$ in (7); hence (13) and (14) contradict (7). Therefore the inequality sign holds in $\left(8_{1}\right)$.

5. Proof of Theorem 1. To establish Theorem 1, it suffices (cf. (5) and Lemma 1) to exhibit a particular transformation of the general type (6) with the following property: if $x \neq W$ is a positive solution of (2), a finite number of applications (preferably in a prescribed order) of the transformation carries $x$ into $W$. We next present a convenient such transformation, of the preferred type.

In $x$, let the elements $x_{p}$ which exceed their correspondents $W_{p}$ of $W$ (cf. (3)) -be denoted by $x_{q_{1}}, x_{q_{2}}, \cdots$, where $q_{1}<q_{2}<\cdots$, and let the $x_{p}$ which are less than $W_{p}$ be designated $x_{1}, x_{2 q}, \cdots$, where ${ }_{1} q<{ }_{2} q<\cdots$. Then we define our transformation of $x$ into a new solution $x^{\prime}$ of (2) by $t_{1}$ or $t_{2}$ :

$$
\begin{aligned}
& \left(t_{1}\right) \quad x_{p}^{\prime}=x_{p}\left(p \neq q_{1}, q\right), \quad x_{q_{1}}^{\prime}=M, Q\left(1 / x^{\prime}\right)=Q(1 / x) ; \\
& \left(t_{2}\right) \quad \text { " } \quad, x_{1 q}^{\prime}=M, \quad \text { " } \quad \text { " , }
\end{aligned}
$$

according as $t_{1}$ defines $x_{1}{ }^{\prime}$ to be not greater than $M$ or greater than $M$, respectively. 
Whether $t_{1}$ or $t_{2}$ of (15) is used, it is obvious that $x_{1 q}<x_{1 q}{ }^{\prime} \leqq M$ and $x_{q_{1}}$ $>x_{q_{1}}{ }^{\prime} \geqq M$. Hence transformation (15) is of the type (6) (because each transformation keeps $(n-2)$ of the $x$ 's fixed and increases (decreases) an $x_{i}<M$ $\left(x_{j}>M\right)$ to a value $\left.x_{i}^{\prime} \leqq M\left(x_{j}^{\prime} \geqq M\right)\right)$, and $x^{\prime}$ obviously contains at least one more element of solution $W$ than does $x$. If $x^{\prime} \neq W$, let $x_{q_{1}{ }^{\prime}}^{\prime}, x_{q_{2}{ }^{\prime}}^{\prime}, \cdots$ be the elements of $x^{\prime}$ which exceed $M$, where $q_{1}^{\prime}<q_{2}^{\prime}<\cdots$, and let $x_{1 q^{\prime}}^{\prime}, x_{2 q^{\prime}}^{\prime}, \cdots$ be the elements of $x^{\prime}$ which are less than $M$ where ${ }_{1} q^{\prime}<_{2} q^{\prime}<\ldots$. Then we may evidently repeat our transformation (15) with $x^{\prime \prime}, x^{\prime}, q^{\prime}$ in the place of $x^{\prime}, x, q$, respectively, and obtain a set $x^{\prime \prime}$ which contains at least one more element of solution $W$ than does $x^{\prime}$; etc., with the same method of repeating the transformation until $x$ is carried into $W$. Hence transformation (15) is of the type desired, and Theorem 1 is true.

\section{Part 2. A general approach to OUR THEORY OF MAXIMUM NUMBers}

6. An equivalent of Lemma 1 , and a transformation which increases $P(x)$. The following lemma, which is equivalent to Lemma 1 , is fundamental in our theory of maximum numbers.

Lemma 1a. With $i$ and $j$ equal to distinct positive integers, each $\leqq n$, the number of variables in (2), if $x_{i}, x_{j}, \alpha, \beta$ are positive numbers such that $\alpha<x_{i} \leqq x_{j}$; if $p,(q, s), A_{p}, B_{q s}$ have the meanings here that they have in (4); and if

$$
\begin{aligned}
& \sum_{(p)} A_{p}\left[\left(x_{i}-\alpha\right)^{-p}+\left(x_{j}+\beta\right)^{-p}\right] \\
+ & \sum_{(q s)} B_{q s}\left(x_{i}-\alpha\right)^{-q}\left(x_{j}+\beta\right)^{-q}\left[\left(x_{i}-\alpha\right)^{-s}+\left(x_{j}+\beta\right)^{-s}\right]=Q(1 / x),,^{*}
\end{aligned}
$$

then

$$
x_{i} x_{j} \leqq\left(x_{i}-\alpha\right)\left(x_{j}+\beta\right),\left(x_{i}^{h}+x_{j}^{h}\right)<\left[\left(x_{i}-\alpha\right)^{h}+\left(x_{j}+\beta\right)^{h}\right],
$$

where $h$ is a positive integer. Furthermore the equality sign holds in $\left(17_{1}\right)$ if, and only if, $Q(1 / x)$ is a polynomial in $\left(x_{1} x_{2} \cdots x_{n}\right)^{-1}$.

The transformation. If $x$ is a positive solution of any given equation of type (2), in which we have supposed that $n>1$, there exist in $x$ two numbers $x_{i}, x_{j}$ such that $x_{i} \leqq x_{1}$. Hence by a transformation of the type

$$
x_{p}^{\prime}=x_{p} \quad(p \neq i, j), x_{i}^{\prime}=x_{i}-\alpha, \quad x_{j}^{\prime}=x_{j}+\beta,
$$

where $x_{i}, x_{j}, \alpha, \beta$ are as they are required to be in Lemma 1a, we obtain for the given equation a positive solution $x^{\prime}$ such that $P(x)<P\left(x^{\prime}\right)$ except in the special case, in which $P(x)=P\left(x^{\prime}\right)$. In subsequent sections of this paper we shall not deal with an equation of type (2) in which $Q(1 / x)$ is a polynomial 
in $\left(x_{1} x_{2} \cdots x_{n}\right)^{-1}$ since such equations are of very little interest; hence we shall not need to consider the special case.

From the last paragraph it is evident that $P(x)$ does not attain a maximum value on the positive solutions of any given equation of type (2) in which $Q(1 / x)$ is not a polynomial in $\left(x_{1} x_{2} \cdots x_{n}\right)^{-1}$; hence for equations (2) in which $Q(1 / x)$ is not such a polynomial, we must not admit all of the positive solutions.

7. The type of solution that we are to study. In considering the case $r=1$ of equation (1), Curtiss and Takenouchi admitted all solutions $x$ in which (i) $x_{1}, x_{2}, \cdots, x_{n-1}$ are positive integers and (ii) $x_{1} \leqq x_{2} \leqq \cdots \leqq x_{n}$. Such solutions of any given equation include all of its positive integral solutions for which (ii) holds and perhaps other solutions, ${ }^{*}$ and will be referred to as $E$ solutions (extended solutions). One naturally asks the following question: for the case $r=1$ of (1), are all positive solutions in which $(n-1)$ of the $x$ 's are integers bounded? That the answer is no is clear from the fact that a solution of the equation $\left(x_{1}^{-1}+x_{2}^{-1}\right)=1$, which is a special case of the equation that we are considering, is given by $\left[\alpha(\alpha-1)^{-1}, \alpha\right]$ where $\alpha$ is any real number $>1$. For the case $r=1$ of (1) the positive solutions in which less than $(n-1)$ of the $x$ 's are integers are of course also unbounded. In as much as Curtiss and Takenouchi have shown that the $E$-solutions of every equation of type (1) for which $r=1$ are bounded, $\dagger$ it is now clear that the $E$-solution was the natural type for them to consider. Now since we have relative to $E$-solutions a theory which will obtain (as we are to show in the sequel) the results mentioned in the first two paragraphs of $\$ 1$, it is obviously desirable that we choose $E$-solutions as the type to study. This we do.

In the next section we shall show (rather point out that Curtiss has proved without observing the fact) that the $E$-solutions of every equation of type (2) are bounded. Then in the rest of Part 2 we shall present certain further facts of interest about $E$-solutions.

8. Proof that the $E$-solutions of every equation of type (2) are bounded. If we can prove that the $E$-solutions of every equation of type (2) that has one or more $E$-solutions are bounded, we shall have reduced the problem of finding all of these $E$-solutions to a finite number of trials. We shall now show that this has been essentially done by Curtiss in an article in which he proved

* For example, the equation $\left(x_{1}^{-1}+x_{2}^{-1}\right)=2 / 7$ has only two positive integral solutions for which (ii) holds, namely $(4,28)$ and $(7,7)$, and has four solutions that satisfy (i) and (ii), namely the two just given and $(5,35 / 3),(6,42 / 5)$.

$\dagger$ If the $E$-solutions of any given equation of type (2) are bounded, there is only a finite number of sets of values $\left(x_{1}, x_{2}, \cdots, x_{n-1}\right)$ that belong to $E$-solutions of the equation, and if $x_{1}, x_{2}, \cdots, x_{n-1}$ are given elements of such an $E$-solution, its $n$th element is uniquely determined. Thus if the $E$-solutions of any equation of type (2) are bounded, they are finite in number. 
that the positive integral solutions of an equation that includes (2) are bounded.* His equation (1), p. 859 of the article just cited, includes our equation (2). The argument which he carried through in arriving at the relations (5), p. 861, is based on the assumptions that $x_{1} \leqq x_{2} \leqq \cdots \leqq x_{n}$ and that $x$ is a positive integral solution of (1), p. 859. However, he did not use the assumption that $x_{n}$ is an integer; the hypotheses which he actually used are precisely (i) and (ii) of $\$ 7$. Consequently his procedure gives the following result: the E-solutions of every equation of type (1), p. 859, are bounded; they have the bounds that are defined by relations (5), p. 861. Consequently the $E$-solutions of equation (2) above are bounded.

In the next section we consider an example in which the number of trials referred to above is small; furthermore, one $E$-solution that is obtained in this example is of particular interest because it has two properties with which we shall be greatly concerned in the sequel.

9. An example of a class of maximum numbers and of an individual maximum number associated with an equation of type (2) that is not elementary symmetric. Suppose that

$$
x_{1}^{-1}+x_{2}^{-1}+\left(x_{1} x_{2}\right)^{-1}+x_{1}^{-2}+x_{2}^{-2}=1 .
$$

The only $E$-solutions that $(19)$ has are $x=v \equiv(3,3)$ and $x=w \equiv\left(2,3+13^{1 / 2}\right)$. To prove that $w$ gives to every polynomial of type $P(x)\left(=P\left(x_{1}, x_{2}\right)\right.$ here, since $Q(1 / x)$ is the left member of (19)) a larger value than does $v$, it suffices to show that if in the notation of Lemma 1a, $\left(x_{i}, x_{j}\right)=\left(x_{1}, x_{2}\right)=(3,3)$ and if $\alpha=1, \beta=13^{1 / 2}$, then (17) holds with $<$ in its first relation. This conclusion follows from Lemma 1a and the fact that in (19) $Q(1 / x)$ is not a polynomial in $\left(x_{1} x_{2}\right)^{-1}$.

Hence every polynomial of the type $P(x)$ just mentioned is maximized (with respect to values that are given to it by $E$-solutions of (19)) by taking $x=w$. Since there are infinitely many such polynomials, we have identified relative to (19) infinitely many maximum numbers. Furthermore, we note that $w$ is the solution to which Kellogg's process $\dagger$ leads, and that since $w_{2}=\left(3+13^{1 / 2}\right)$ is the largest number that appears in either $w$ or $v$, w contains

* Cf. D. R. Curtiss, Classes of Diophantine equations whose positive integral solutions are bounded, Bulletin of the American Mathematical Society, 1929, p. 859.

$\dagger$ Cf. O. D. Kellogg, loc. cit. In obtaining his interesting solution of the equation $\Sigma\left(1 / x_{1}\right)=1$, Kellogg proceeded as follows. He first assigned to $x_{1}$ the smallest (positive integral) value, say $x_{1}=w_{1}(=2)$, that satisfies the inequality $x_{1}^{-1}<1$; then he assigned to $x_{2}$ the smallest value, say $x_{2}=w_{2}(=3)$, such that $\left(w_{1}, w_{2}\right)$ satisfies the inequality $\left(x_{1}^{-1}+x_{2}^{-1}\right)<1$; and he continued minimizing the remaining variables of the set $x_{1}, x_{2}, \cdots, x_{n-1}$ in this order, one at a time, until all of them were fixed, say $\left(x_{1}, x_{2}, \cdots, x_{n-1}\right)=\left(w_{1}, w_{2}, \cdots, w_{n-1}\right)$. It turned out that the value thus determined by the equation $\Sigma\left(1 / x_{1}\right)=1$ for $x_{n} \equiv w_{n}$ was an integer with a remarkable property that is described in Curtiss's article (loc. cit. in third footnote on p. 876). 
the maximum number that exists in any E-solution of (19), while no other $E$ solution of (19) has this property.

10. Kellogg solutions. In the rest of this paper if a solution $x$ of any given equation is obtained by Kellogg's process (of minimizing $x_{1}, x_{2}, \cdots, x_{n-1}$ in this order, one at a time), we shall denote it by $w$ and call it the Kellogg solution of the given equation. Nearly all of the rest of this paper will be devoted to the identification of maximum numbers that we associate with Kellogg solutions of certain elementary symmetric equations which are special cases of (2). Every Kellogg solution with which we shall be concerned is an $E$-solution,* though of course the converse is not the case (cf. first footnote on page 882). After we obtain the Kellogg solution $w$ of a given equation, we attempt to ascertain whether $w$ has the two properties which were described for the solution $\left(3,3+13^{1 / 2}\right)$ in the example of $\$ 9$, namely (I) $w_{n}$ is the largest number that exists in any $E$-solution of the given equation and $w_{n}$ appears in but one such $E$-solution; (II) if $x$ is any $E$-solution except $w$ of the given equation, $P(x)<P(w) . \dagger$ In the cases of some solutions $w$ (of elementary symmetric equations) that we obtain, we are unfortunately unable to determine whether or not they have either of the properties I and II (cf. \$23).

We next present two general lemmas which will be of use in establishing properties I and II for certain solutions $w$ that we are to study in the sequel.

11. Important lemmas. Suppose that when the left member of (2) is expressed as a polynomial in $x_{n}^{-1}$ the resulting equation is

(20) $Q(1 / x) \equiv Q_{0}(1 / x)+Q_{1}(1 / x) \cdot x_{n}^{-1}+Q_{2}(1 / x) \cdot x_{n}^{-2}+\cdots+Q_{\lambda}(1 / x) \cdot x_{n}^{-\lambda}=c$, where the $Q_{p}(1 / x) \equiv Q_{p}\left(1 / x_{1}, 1 / x_{2}, \cdots, 1 / x_{n-1}\right)(p=0,1, \cdots, \lambda)$ are symmetric polynomials in the $x_{t}^{-1}(t=1, \cdots, n-1)$, with no negative coefficient, and where at least one of the $Q_{p}(1 / x), p>0$, is not zero. Then the following lemma is obviously true.

Lemma 2. Suppose there exists an E-solution, say $x=u$, of (20) with the property that if $x$ is any $E$-solution except $u$ of (20),

$$
Q_{p}(1 / x) \leqq Q_{p}(1 / u) \quad(p=0,1, \cdots, \lambda),
$$

the sign $<$ holding for at least one of the specified values of $p$; then it follows that $u_{n}$ is the largest number that exists in any E-solution of (20), and $u_{n}$ appears in but one E-solution of this equation.

* The Kellogg solution of a given equation of type (2) may not be an $E$-solution. For cxample, the Kellogg solution of the equation $\left(x_{1}^{-2}+x_{2}^{-2}\right)=1$ is $w^{\prime}=\left(2,2 / 3^{1 / 2}\right)$, and since $w_{2}^{\prime}<w_{1}^{\prime}$, $w^{\prime}$ is not an $E$-solution.

† It is interesting to note that the Kellogg solution, which obviously exists for every equation of type (2), may be an $E$-solution and yet not have either of the properties I and II. For cxample, the Kellogg solution of the equation $\left(x_{1}^{-1}+x_{2}^{-1}+x_{3}^{-1}\right)=(5 / 10)$ is $u^{\prime}=(4,17,272)$, while $v \equiv(5,9,720)$ is also an $E$-solution of this equation, and here $v_{3}>i_{3},\left(w_{1}+i_{2}+i_{3}\right)>\left(w_{1}+i_{2}+w_{3}\right)$, and $v_{1} i_{2} v_{3}>\pi_{1}{ }_{1} i_{2} i_{3}$. 
Remark. In terms of Lemma 2, we observe that Curtiss's result on Kellogg's Diophantine problem was obtained by showing that with

where

$$
Q(1 / x) \equiv Q_{0}(1 / x)+Q_{1}(1 / x) \cdot x_{n}^{-1}=1,
$$

$$
Q_{0}(1 / x) \equiv x_{1}^{-1}+x_{2}^{-1}+\cdots+x_{n-1}^{-1}, \quad Q_{1}(1 / x) \equiv 1,
$$

Kellogg's solution $w$ is the $u$ of Lemma 2 ; that is, if $x$ is any $E$-solution except $w(\equiv u)$, then $Q_{0}(1 / x)<Q_{0}(1 / w)$ and $Q_{1}(1 / x)=Q_{1}(1 / w)=1$.

Lemma 3. Suppose there exists an E-solution $x=u$ of (2) with the property that if $x$ is any E-solution other than $u$ of (2), it is possible to transform $x$ into $u$ by one or more transformations of type (18), in which the notation of Lemma 1a holds; then it follows that $P(x)<P(u)$.

\section{Part 3. The individual maximum number and the class of maximum} NUMBERS THAT WE ASSOCIATE WITH EQUATION (1)

12. The Kellogg solution of an elementary symmetric equation. Statements of two theorems. With $i \geqq 0$ and $j$ equal to integers, we let $\Sigma_{i, j}(x)$ stand for the $j$ th elementary symmetric function of the $i$ variables $x_{1}, x_{2}, \cdots, x_{i}$; with the (customary) understanding that

$$
\Sigma_{i, j}(x)\left\{\begin{array}{l}
\equiv 0 \text { when } i<j \text { and also when } j<0 ; \\
\equiv 1 \text { when } j=0 .
\end{array}\right.
$$

The equation whose Kellogg solution we now desire is

$$
\begin{aligned}
\Sigma_{n, r}(1 / x)+\lambda_{r+1} \Sigma_{n, r+1}(1 / x)+ & \lambda_{r+2} \Sigma_{n, r+2}(1 / x)+\cdots \\
& +\lambda_{s} \Sigma_{n, s}(1 / x)=b / a, \quad a \equiv[(c+1) b-1],{ }^{*}
\end{aligned}
$$

in which $r, s, n$ are any positive integers such that $r<s \leqq n ; b, c$ are any positive integers; and the $\lambda_{p}(p=r+1, r+2, \cdots, s)$ are integers $\geqq 0$. In obtaining the solution in question, we first express the $\Sigma_{n, p}(1 / x)$ of (21) by means of the following identities, which are convenient for our purposes:

$$
\begin{aligned}
& \Sigma_{n, r}(1 / x) \equiv \frac{1}{x_{1} x_{2} \cdots x_{r}}+\sum_{p=r}^{n-1} \frac{1}{x_{p+1}} \cdot \frac{\Sigma_{p, p-r+1}(x)}{x_{1} x_{2} \cdots x_{p}} \\
& \Sigma_{n, j}(1 / x) \equiv \sum_{p=r}^{n-1} \frac{1}{x_{p+1}} \cdot \frac{\Sigma_{p, p-j+1}(x)}{x_{1} x_{2} \cdots x_{p}} \quad(j=r+1, r+2, \cdots, s) .
\end{aligned}
$$

* By taking $a$ in this way, we generalize a problem of Takenouchi (loc. cit. in third footnote on p. 876), and we have in (21) an equation whose Kellogg solution is a solution in positive integers (cf. (23)). With all symbols of (21) except $a$ as they are defined just below (21), our choice of $a$ is the only one $\neq 1$ that we have found with the property that the Kellogg solution of the resulting equation (21) is a solution in positive integers. 
Then we find that (21) is equivalent to the following equation:

$$
\begin{aligned}
& \frac{1}{x_{1} x_{2} \cdots x_{r}} \\
& +\sum_{p=r}^{n-1} \frac{1}{x_{p+1}}\left[\frac{\Sigma_{p, p-r+1}(x)+\lambda_{r+1} \Sigma_{p, p-r}(x)+\cdots+\lambda_{s} \Sigma_{p, p-s+1}(x)}{x_{1} x_{2} \cdots x_{p}}\right]=\frac{b}{a},
\end{aligned}
$$

where $a \equiv[(c+1) b-1]$ is as it was defined for equation (1). Consider now the set of numbers $x=w$, where

$$
\begin{aligned}
w_{p}= & 1 \quad(p=1, \cdots, r-1), \quad w_{r}=c+1, \\
w_{p+1}= & a\left[\Sigma_{p, p-r+1}(w)+\lambda_{r+1} \Sigma_{p, p-r}(w)+\lambda_{r+2} \Sigma_{p, p-r-1}(w)+\cdots\right. \\
& \left.+\lambda_{s} \Sigma_{p, p-s+1}(w)\right]+1 \quad(p=r, \cdots, n-2), \\
w_{n}= & a\left[\Sigma_{n-1, n-r}(w)+\lambda_{r+1} \Sigma_{n-1, n-r-1}(w)+\lambda_{r+2} \Sigma_{n-1, n-r-2}(w)+\cdots\right. \\
& \left.+\lambda_{s} \Sigma_{n-1, n-8}(w)\right] .
\end{aligned}
$$

To prove that $w$ is a solution of (22), we replace $x$ in (22) by $w$ and observe that

$$
\begin{aligned}
& \frac{1}{w_{p+1}}\left[\frac{\Sigma_{p, p-r+1}(w)+\lambda_{r+1} \Sigma_{p, p-r}(w)+\lambda_{r+2} \Sigma_{p, p-r-1}(w)+\cdots+\lambda_{s} \Sigma_{p, p-s+1}(w)}{w_{1} w_{2} \cdots w_{p}}\right] \\
& =\frac{w_{p+1}-1}{a w_{1} w_{2} \cdots w_{p+1}}=\frac{1}{a w_{1} w_{2} \cdots w_{p}}-\frac{1}{a w_{1} w_{2} \cdots w_{p+1}} \quad(p=r, \cdots, n-2),
\end{aligned}
$$

while

$$
\begin{gathered}
\frac{1}{w_{n}}\left[\frac{\Sigma_{n-1, n-r}(w)+\lambda_{r+1} \Sigma_{n-1, n-r-1}(w)+\lambda_{r+2} \Sigma_{n-1, n-r-2}(w)+\cdots+\lambda_{s} \Sigma_{n-1, n-s}(w)}{w_{1} w_{2} \cdots w_{n-1}}\right] \\
=\frac{1}{a w_{1} w_{2} \cdots w_{n-1}},
\end{gathered}
$$

so that from (22) we obtain

$$
\begin{aligned}
& \frac{1}{w_{1} w_{2} \cdots w_{r}}+\left(\frac{1}{a w_{1} w_{2} \cdots w_{r}}-\frac{1}{a w_{1} w_{2} \cdots w_{r+1}}\right) \\
& +\left(\frac{1}{a w_{1} w_{2} \cdots w_{r+1}}-\frac{1}{a w_{1} w_{2} \cdots w_{r+2}}\right) \\
& +\cdots+\left(\frac{1}{a w_{1} w_{2} \cdots w_{n-2}}-\frac{1}{a w_{1} w_{2} \cdots w_{n-1}}\right)+\frac{1}{a w_{1} w_{2} \cdots w_{n-1}} \\
& =\frac{1}{w_{1} w_{2} \cdots w_{r}}+\frac{1}{a w_{1} w_{2} \cdots w_{r}}=\frac{a+1}{a w_{1} w_{2} \cdots w_{r}}=\frac{b}{a} ;
\end{aligned}
$$


the last two equalities following readily from (23) and the definition of $a$.

That solution $w$ is the Kellogg solution of (21) can be seen from the following three statements: (i) in (23), $w_{1}, w_{2}, \cdots, w_{r-1}$ are all equal to unity. so that each of these elements has as small a value as it could have in the Kellogg solution of any equation of type (2); (ii) $w_{r}=(c+1)$ exceeds the greatest integer in $(a / b)$ by unity, and on account of (i) it is apparent that $w_{r}$ is the $r$ th Kellogg number for (21); (iii) since statements (i) and (ii) are true, comparison of the expressions for $w_{p+1}(p=r, \cdots, n-2)$, and $w_{n}$, in (23), shows that $w_{r+1}, \cdots, w_{n-1}$ are the $(r+1) s t, \cdots,(m-1)$ st Kellogg numbers, respectively, for (21).

Unfortunately our method of identifying maximum numbers does not apply to the general equation (21). Our major purpose in the rest of this paper is to prove the following theorems.

THEOREM 2. The largest number that exists in any E-solution of the equation

$$
\Sigma_{n, r}(1 / x)=b / a, \quad a \equiv[(c+1) b-1]
$$

(an equivalent of equation (1)), in which every symbol that appears is as it was defined for (21), is the $w_{n}$ of the following equations [cf. (23)]:

$$
\begin{array}{rlrl}
w_{p} & =1 & (p=1, \cdots, r-1), & w_{r}=c+1, \\
w_{p+1}=a \Sigma_{p, p-r+1}(w)+1 & (p=r, \cdots, n-2), & w_{n}=a \Sigma_{n-1, n-r}(w) .
\end{array}
$$

Furthermore, $w_{n}$ appears in but one E-solution of (25).*

THEOREM 3. If $x \neq w$ is any E-solution of (25), then $P(x)<P(w)$, where $P(x)$ is as it was defined in $\$ 2$, with the understanding that here the $Q(1 / x)$ of $\$ 2$ is the left member of (25).

* That (25) may have many $E$-solutions is shown by the following example. Suppose that $n=5$ and $r=b=c=1$ (so that $a=1$ ). Then (25) becomes

(A)

$$
\Sigma_{5,1}(1 / x)=1 \text {, }
$$

whose Kellogg solution is (cf. (26)) $w_{1}=2, w_{2}=3, w_{3}=7, w_{4}=43, w_{5}=1806$. If we take $x_{1}=2, x_{2}=3$, $x_{3}=7$, and $x_{4}=x_{5} \equiv x^{\prime}$ in (A), we find that the resulting equation has the solution $x^{\prime}=84=2\left(w_{4}-1\right)$. Consequently (A) has the following $\left(w_{4}-1\right) E$-solutions in which $x_{p}=w_{p}(p=1,2,3):[2,3,7, \alpha, 42 \alpha /$ $(\alpha-42)], \alpha=43,44, \cdots, 84$.

Similarly, by considering the equation $\Sigma_{n, 1}(1 / x)=1$ and its Kellogg solution (cf. (26) with $r=a=c=1)$, one can show that this equation has $\left(w_{n-1}-1\right) E$-solutions in each of which $x_{p}=w_{p}$ $(p=1,2, \cdots, n-2)$.

Relative to the general equation (25) and its Kellogg solution $w$ of (26), one can also obtain an interesting result, as follows. If we take

and $x_{n-1}=x_{n} \equiv x^{\prime}$ in (25), the resulting equation in $x^{\prime}$ will have exactly one positive root, say $x^{\prime}=R$, where $w_{n-1} \leqq R \leqq w_{n}$. Let $u$ stand for the greatest integer in $R$. Then it follows that (25) has exactly as many $E$-solutions in which (B) holds as there are positive integers $v$ such that $w_{n-1} \leqq v \leqq u$. 
In $\$ 22$, we shall show why our method of attack does not suffice to obtain for (21) results that are analogous to Theorem $i(i=2,3)$. In $\$ 23$, we shall state relative to certain cases of (21) in which the $\lambda$ 's are not all equal to zero further results (Theorem 4 and Theorem 5 ) that can be obtained by the method which we use in proving Theorem $i(i=2,3)$; and we shall exhibit the Kellogg solution of, and state theorems which are analogous to Theorem $i(i=4$, 5) for, a rather general elementary symmetric equation that differs from (21).

13. Important inequalities. We first obtain a set of equalities which led us to consider the inequalities in question. If $k$ is a positive integer $\leqq(n-r)$, the sum of the first $2 k$ terms of (24) (in which two terms are counted for each parenthesis) is $b / a$; and the sum of the first term and the first $(p-r), r \leqq p$ $\leqq(n-1)$, parentheses of $(24)$ is $\left(b w_{1} w_{2} \cdots w_{p}-1\right)\left(a w_{1} w_{2} \cdots w_{p}\right)^{-1}$. Now since (24) was obtained by setting $x=w$ in (22), an equivalent of (21), it is evident that

$$
\begin{aligned}
& \Sigma_{p, r}(1 / w)+\lambda_{r+1} \Sigma_{p, r+1}(1 / w)+\lambda_{r+2} \Sigma_{p, r+2}(1 / w)+\cdots+\lambda_{s} \Sigma_{p, s}(1 / w) \\
& \quad=\left(b w_{1} w_{2} \cdots w_{p}-1\right) /\left(a w_{1} w_{2} \cdots w_{p}\right) \quad(p=r, r+1, \cdots, n-1) .
\end{aligned}
$$

If $\lambda_{t}=0(t=r+1, \cdots, s)$, it follows from (27) that the $w$ of (26) satisfies the following equations, which we set out to obtain:

$$
\Sigma_{p, r}(1 / w)=\left(b w_{1} w_{2} \cdots w_{p}-1\right) /\left(a w_{1} w_{2} \cdots w_{p}\right)(p=r, r+1, \cdots, n-1) .
$$

In the sequel except where the contrary is stated $w$ will stand for the solution (26).

Equalities (28) lead one to inquire as to the validity of the following statement, which we shall prove to be true: if for (25) $X$ is any $E$-solution $\neq w$, then

$$
\begin{array}{r}
\Sigma_{p, r}(1 / X) \leqq\left(b X_{1} X_{2} \cdots X_{p}-1\right) /\left(a X_{1} X_{2} \cdots X_{p}\right) \\
\quad(p=r, r+1, \cdots, n-1) .
\end{array}
$$

An equivalent of the equation that results when $x$ of (25) is replaced by $Y$ is

$$
\begin{aligned}
& \left(1 / X_{p+1}\right) \Sigma_{p, r-1}(1 / X)+\left(1 / X_{p+2}\right) \Sigma_{p+1, r-1}(1 / X)+\cdots+\left(1 / X_{n}\right) \Sigma_{n-1, r-1}(1 / X) \\
& \quad=\left[(b / a)-\Sigma_{p, r}(1 / X)\right]=\left[b X_{1} X_{2} \cdots X_{p}-a \Sigma_{p, p-r}(X)\right] /\left(a X_{1} X_{2} \cdots X_{p}\right) .
\end{aligned}
$$

By hypothesis, $X_{1}, X_{2}, \cdots, X_{p}$, the first $p, r \leqq p<n$, numbers of an $E$ solution, are positive integers. Therefore both numerator and denominator of the last fraction displayed are positive integers. Hence

$$
\left[(b / a)-\Sigma_{p, r}(1 / X)\right] \geqq\left(a X_{1} X_{2} \cdots X_{p}\right)^{-1},
$$

and (29) holds. 
In the sequel except where the contrary is stated $X$ will be understood to be any $E$-solution of (25) except $w$.

The importance of the case $p=(n-1)$ of both (28) and (29) can be seen from the following two facts: first, an equivalent of equation (25) is

$$
\Sigma_{n-1, r}(1 / x)+\left(1 / x_{n}\right) \Sigma_{n-1, r-1}(1 / x)=b / a ;
$$

second, to prove Theorem 2 it suffices to show that

$$
\begin{aligned}
\Sigma_{n-1, r}(1 / X) & \leqq \Sigma_{n-1, r}(1 / w) \text { for } 1 \leqq r<n ; * \\
\Sigma_{n-1, r}(1 / X) & <\Sigma_{n-1, r}(1 / w) \text { for } r=1 ; \\
\Sigma_{n-1, r-1}(1 / X) & <\Sigma_{n-1, r-1}(1 / w) \text { for } 1<r<n .
\end{aligned}
$$

Our major difficulty is in establishing (30). After this is done by our method, (31) and (32) will easily follow, as will also Theorem 3.

14. The nature of the induction for (30). Lemmas 4 and 5. From (28) and (29) one sees that if (30) is not true then $X_{1} X_{2} \cdots X_{n-1}>w_{1} w_{2} \cdots w_{n-1}$. In the induction that we are to make in proving (30), we shall consider the following more general fact: if for any one of the values of $p$ in (29),

then for this $p$

$$
\Sigma_{p, r}(1 / X)>\Sigma_{p, r}(1 / w),
$$

$$
\frac{b X_{1} X_{2} \cdots X_{p}-1}{a X_{1} X_{2} \cdots X_{p}} \geqq \Sigma_{p, r}(1 / X)>\frac{b w_{1} w_{2} \cdots w_{p}-1}{a w_{1} w_{2} \cdots w_{p}},
$$

so that $X_{1} X_{2} \cdots X_{p}>w_{1} w_{2} \cdots w_{p}$. Let $x_{1} \ldots p$ (to be read $x 1$ to $p$ ) stand for $x_{1}, x_{2}, \cdots, x_{p}$ in this order; the small letter $x$ being used here because the notation which we are defining is to apply (not only to $X$ but also) to every set of numbers that we consider. To prove (30), we shall proceed as follows. We suppose that there exist one or more positive integers $p \leqq(n-1)$ for which

$$
X_{1} \ldots p \neq w_{1} \ldots p \text { and } \Sigma_{p, r}(1 / X)>\Sigma_{p, r}(1 / w)
$$

(so that $X_{1} X_{2} \cdots X_{p}>w_{1} w_{2} \cdots w_{p}$ ); and let $k$ be the smallest such integer $p$. Then we shall reach a contradiction by showing that if the last two displayed statements hold when $p=k$, then $w_{1} w_{2} \cdots w_{k}>X_{1} X_{2} \cdots X_{k}$ (cf. (51), \$16).

The definition below enables one to describe the above induction briefly. In this definition (and indeed throughout our discussion of maximum numbers) we suppose that $x_{p} \geqq 1(p=1,2, \cdots, n)$ in every set $x$ that we consider.

Definition. Let $\lambda$ be a fixed positive integer such that $r \leqq \lambda \leqq n$, where $r$

* Were we to prove (31), (32) and the relation that is obtained from (30) by merely replacing $1 \leqq r<n$ by $1<r<n$, Theorem 2 would follow. However, we find it convenient to prove (30) before proving (31). 
and $n$ are as they are defined for (25). We shall call $x_{1} \ldots$ p a set $\sigma$ (relative to the $w$ of (26)) if, and only if, $\Sigma_{p, r}(1 / x) \leqq \Sigma_{p, r}(1 / w)$ for every positive integer $p$ such that $r \leqq p \leqq \lambda$. We shall call $x_{1} \cdots(\lambda+1)$ a set $\tau$ if, and only if, $\lambda$ is a positive integer such that $r \leqq \lambda \leqq(n-2)$ and $x_{1} \ldots(\lambda+1)$ is not, and $x_{1} \ldots \lambda$ is, a set $\sigma$.

Remark. The number of elements in a set $\sigma[\tau]$ is at least $r[r+1]$ and at most $n[n-1]$.

It is evident now that to prove (30) it suffices to show that for (25) every $E$-solution $\neq w$ is a set $\sigma$ (for which $\lambda=n$ ).

In proving (30) we shall use certain terminology that we have not yet defined. However, before introducing that, we present here two lemmas which one now logically desires. The first, Lemma 4 , states that $X_{1} \ldots$ r is a set $\sigma$ and thus begins the induction for (30). The second, Lemma 5 , has a significance which may be described as follows. Since $X$ is by its definition an $E$-solution $\neq w, X$ contains two elements $X_{q_{1}}$ and $X_{1 q}$ (such that $X_{q_{1}}>w_{q_{1}}$ and $X_{1 q}<w_{1 q}$ ). Lemma 5 states that $q_{1}<_{1} q$. Then since $X$ is an $E$-solution, $X_{q_{1}} \leqq X_{1}$. Consequently (Lemma 1a) it is possible to apply to $X$ at least one transformation of the type (18) and obtain a new solution $X^{\prime}$ such that $P(X)<P\left(X^{\prime}\right)$; the possibility of the equality of $P(X)$ and $P\left(X^{\prime}\right)$ being excluded by the fact that $Q(1 / x)\left(\equiv \Sigma_{n, r}(1 / X)\right.$ here, in which $\left.n>r\right)$ is not a polynomial in $\left(X_{1} X_{2} \ldots\right.$ $\left.X_{n}\right)^{-1}$.

LEMMA 4. $X_{1} \ldots$ is a set $\sigma$.

By hypothesis, $X_{1} \ldots r$ consists of $r(<n)$ positive integers $X_{1}, X_{2}, \ldots$, $X_{r}$ or one positive integer $X_{1}$ when $r=1$, and $\left(X_{1} X_{2} \cdots X_{r}\right)^{-1}<b[(c+1) b$ $-1]^{-1}$ (cf. (25)). Therefore $X_{1} X_{2} \cdots X_{r} \geqq(c+1)$. Whence $\Sigma_{\tau, \tau}(1 / X)$ $\leqq \Sigma_{r, r}(1 / w)$ (cf. (26)), and $X_{1} \ldots$, is a set $\sigma$.

Thus when $r=(n-1)$, every $E$-solution of $(25)$ is a set $\sigma$.

LEMMA 5. If $x_{1} \ldots k \neq w_{1} \ldots k$ stands for (i) $X_{1} \ldots k(r \leqq k \leqq n)$, (ii) any set $\sigma,{ }^{*}$ or (iii) any set $\tau$ with at least one element larger than, and at least one element less than, its correspondent $\dagger$ in $w_{1} \ldots k$; then the smallest integer $t, 1 \leqq t \leqq k$, for which $x_{t} \neq w_{t}$ is such that $x_{t}>w_{t}$.

(i). By hypothesis $X_{1} \ldots k \neq w_{1} \ldots k$ and $X_{1} \ldots k$ is the ordered set $X_{1}$, $X_{2}, \cdots, X_{k}$ of an $E$-solution of (25). Hence with hypothesis (i) holding, our conclusion is a consequence of the fact that $w_{1} \ldots k \equiv\left(w_{1}, w_{2}, \cdots, w_{k}\right)$ is a part of the Kellogg solution of (25) (cf. second footnote on page 883 and $\$ 10$ ).

(ii). Suppose that the smallest positive integer $t$ for which $x_{t} \neq w_{t}$ is such

* The values which $k$ can assume in (ii) and (iii) can be seen from the Definition of the present section.

$\dagger$ If $x_{i}$ and $y_{j}$ are elements of the sets $x_{1} \ldots k$ and $y_{1} \ldots k$, respectively, $x_{i}$ and $y_{j}$ will be called corresponding elements of these sets if, and only if, $i=j$. 
that $x_{t}<w_{t}$. Then $x_{p}=w_{p}(p=1,2, \cdots, t-1)$ and $x_{t}<w_{t}$, where $t \geqq r$ (cf. (26) and our assumption that every element which we consider $\geqq 1)$. Therefore $\Sigma_{t, r}(1 / x)>\Sigma_{t, r}(1 / w)$, which contradicts our hypothesis that $x_{1} \ldots t$ is a set $\sigma$. Hence $x_{t}>w_{t}$.

(iii). Since $x_{1} \ldots k$ is a set $\tau, x_{1} \ldots(k-1)$ is a set $\sigma$. By hypothesis $x_{1} \ldots k$ contains at least two elements that differ from their correspondents in $w_{1} \ldots k$. Therefore $x_{1} \ldots(k-1) \neq w_{1} \ldots(k-1)$. Hence our argument relative to hypothesis (ii) can be applied to obtain the desired conclusion for the present case.

15. Classification and transformation of elements. In this section we give our classification and transformation of $X_{1} \ldots \nu$ where $\nu$ is an integer such that $r \leqq \nu \leqq n$ and $X$ is of course the arbitrary $E$-solution $\neq w$ that we are considering.

Classification. If $X_{1} \ldots \nu \neq w_{1} \ldots \nu$, there is at least one positive integer $p \leqq \nu$ such that $X_{p} \neq w_{p}$. For all such integers $p$, we divide the numbers $X_{p}$ into two mutually exclusive classes $A$ and $B$, which are as follows. Class $A[B]$ consists of all elements $X_{p}$ of $X_{1} \ldots \nu$ for which $X_{p}>w_{p}\left[X_{p}<w_{p}\right]$. To distinguish between the elements of class $A$ and those of class $B$, we employ the following subscript notation. The elements of class $A[B]$ will be designated $X_{q_{1}}, X_{q_{2}}, X_{q_{8}}, \cdots$, where $q_{1}<q_{2}<q_{3}<\cdots\left[X_{19}, X_{2 q}, X_{8 q}, \cdots\right.$, where $\left.{ }_{1} q<_{2} q<_{3} q<\cdots\right]$. If $X_{1} \ldots{ }_{\nu}=w_{1} \ldots \nu$, we shall say that $A$ and $B$ are vacuous classes (without an element). If $X_{1} \ldots \neq \neq w_{1} \ldots \nu$, it may be that either or neither of the classes $A, B$ is vacuous.

Transformation. Suppose $X_{1} \ldots \nu$ contains at least one element of each of the classes $A$ and $B$. Then we define our transformation of $X_{1} \ldots \nu$ into a new set $X_{1}^{\prime} \ldots$ by $t_{3}$ or $t_{4}$ (cf. (15)),

$$
\begin{gathered}
\left(t_{3}\right) \quad X_{p}^{\prime}=X_{p}\left(p \neq q_{1,1} q, p \leqq \nu\right), X_{q_{1}{ }^{\prime}}=w_{q_{1}}, \Sigma_{\nu, r}\left(1 / X^{\prime}\right)=\Sigma_{\nu, r}(1 / X) ; \\
\left(t_{4}\right) \quad \text { " } \quad \text { " } \quad X_{1{ }^{\prime}}=w_{1 q},
\end{gathered}
$$

according as $t_{3}$ requires $X_{1 q}^{\prime}$ to be no greater than $w_{1 q}$ or greater than $w_{1 q}$, respectively.

In case $t_{3}$ defines $X_{1 q}^{\prime}$ to be equal to $w_{1 q}, t_{3}$ and $t_{4}$ are the same transformation, and $X_{1}^{\prime} \ldots \nu$ contains exactly two more elements of the set $w_{1} \ldots \nu$ than does $X_{1} \ldots \nu$. Whether $t_{3}$ or $t_{4}$ is used, $X_{1}^{\prime} \ldots \nu$ contains at least one more element of $w_{1} \ldots$ than does $X_{1} \ldots$.

In the sequel a set $X_{1} \ldots \nu$ with at least one element of each of the classes $A, B$ will be called transformable (by (33)).

Remark. Since $X_{p} \geqq 1(p=1,2, \cdots, n)$, it follows from (26) that ${ }_{1} q \geqq r$. Then since (33) decreases $X_{q_{1}}$ (increases $\left.X_{1 q}\right)$ to a value $\geqq w_{q_{1}}\left(\leqq w_{1 q}\right)$, it follows that $X_{p}^{\prime} \geqq 1$, which accords with our previous agreement concerning the magnitudes of elements $x_{p}$ that we consider (cf. the last paragraph before the Definition in §14). 
16. Lemmas $6,7,8$; second step of the induction described for (30) in $\$ 14$. $^{*}$ We have proved that $X_{1} \ldots$ is a set $\sigma$ (Lemma $\left.4, \S 14\right)$, and thus established (30) for the case $r=(n-1)$. Suppose now that $X_{1} \ldots k$ is a set $\tau$, so that $(r+1) \leqq k \leqq(n-1)$. To the contradiction of this assumption we shall devote a large part of the sequel. In making the first step of the argument in question, we shall employ Lemma 6 below, which includes information that will be of interest after it is shown that $X_{1} \ldots r(r<k \leqq n)$ is a set $\sigma$.

LEMma 6. (i) If $X_{1 \ldots k}$ is a set $\tau \dagger, X_{1} \ldots k$ is transformable. (ii) If $X_{1} \ldots k$ is a set $\tau$, or a transformable set $\sigma$ for which $r<k \leqq n$, and if $t$ is a positive integer, application of (33) with $\nu=k$ to $X_{1} \ldots k$ yields a set $X_{1}^{\prime} \ldots k$ such that

$$
\begin{aligned}
X_{q_{1}} X_{1 q} & <X_{q_{1}} X_{1}{ }^{\prime},\left[X_{q_{1}}{ }^{t}+X_{1}{ }^{t}\right]<\left[\left(X_{q_{1}}{ }^{\prime}\right)^{t}+\left(X_{1}{ }^{\prime}\right)^{t}\right] ; \\
\Sigma_{p, r}\left(1 / X^{\prime}\right) & \leqq \Sigma_{p, r}(1 / w) \text { for } p=r, \cdots,{ }_{1} q-1 ; \\
& =\Sigma_{p, r}(1 / X) \text { for } r=1 \text { and } p={ }_{1} q, \cdots, k-1 ; \\
& <\Sigma_{p, r}(1 / X) \text { for } r>1 \text { and } p={ }_{1} q, \cdots, k-1 ; \\
& =\Sigma_{p, r}(1 / X) \text { for } p=k .
\end{aligned}
$$

Remark. If we prove part (i) of Lemma 6, it will then be evident from the hypothesis of part (ii) that $X_{1} \ldots k$ is surely transformable. Then if we establish relations (35) to (38) inclusive, it will follow from the Definition of $\$ 14$ that if $X_{1} \ldots k$ is a set $\tau$ or a transformable set $\sigma$, then $X_{1}^{\prime} \ldots k$ is a set $\tau$ or a set $\sigma$, respectively.

Proof of (i). By hypothesis, $X$ is an $E$-solution $\neq w$ and so the smallest integer $i$ for which $X_{i} \neq w_{i}$ is $q_{1}$ (cf. Lemma $5, \S 14$ ). Hence $X_{1 \ldots k}$ contains $X_{q_{1}}$ if it contains $X_{1 q}$. Being a set $\tau, X_{1} \ldots k$ contains $X_{1 q}$. Therefore $X_{1 \ldots k}$ is transformable.

Proof of (ii). Equation (38) is true because it is identical with the case $\nu=k$ of the last equation of both $t_{3}$ and $t_{4}$ in (33). Hence we only need to prove relations (34) to (37) inclusive. We begin this task presently.

Proof of (34). From (i) and our hypothesis, $X_{1 \ldots k}$ is transformable, so that $X_{1} \ldots k$ contains $X_{q_{1}}$ and $X_{1}$; and, by Lemma 5, $q_{1}<_{1} q$. Now since the elements of an $E$-solution are arranged in increasing order (when they are written in the order of increasing subscripts), $X_{q_{1}} \leqq X_{1 q}$. Hence relations (34) follow from Lemma 1a, in which we are taking $Q(1 / x)$ to be $\Sigma_{k, r}(1 / X)$, with $r<k$ (cf. footnote (20)), so that $\Sigma_{k, r}(1 / X)$ is not a polynomial in $\left(X_{1} X_{2} \cdots X_{k}\right)^{-1}$.

* We regard Lemma 4 as the first step of this induction.

$\dagger$ The hypotheses of (i) and (ii) in Lemma 6 insure that $r<k$ (cf. the Remark just after the Definition in \$14) so that $\Sigma_{k, r}(1 / X)$ is not a polynomial in $\left(X_{1} X_{2} \cdots X_{r}\right)^{-1}$. It is this fact that enables us to prove (34 $)$ rather than the weaker relation $X_{q_{1}} X_{1 q}=X_{q_{1}}^{\prime} X_{1}^{\prime}$, which, if it held, would prevent our concluding that $P(X)<P\left(X^{\prime}\right)$. 
Proof of (35). From the Remark in the last paragraph of $\$ 15$, we know that ${ }_{1} q \geqq r$. If ${ }_{1} q=r$, we shall say that (35) is vacuously true. If ${ }_{1} q>r, X_{p} \geqq w_{p}$ $\left(p=1, \cdots,{ }_{1} q-1\right)$, by the definition of ${ }_{1} q$ (as the smallest subscript of any element of class $B$ in $X$ ); then from the nature of (33) and the fact that $q_{1}<{ }_{1} q, X_{p}^{\prime} \geqq w_{p}$. Hence (35) holds.

Proof of (36). Since $q_{1}<{ }_{1} q \leqq(k-1)$, if $k<3$ the values of $p$ in (36) form a vacuous set. Suppose $3 \leqq k(\leqq n)$. Whether $t_{3}$ or $t_{4}$ of (33), with $\nu=k$, is used,

$$
X_{p}^{\prime}=X_{p} \quad\left(p \neq q_{1}, 1 q, \quad p \leqq k\right), \quad \Sigma_{k, 1}\left(1 / X^{\prime}\right)=\Sigma_{k, 1}(1 / X) .
$$

Consequently $\left[\left(X_{q_{1}}^{\prime}\right)^{-1}+\left(X_{1 q}^{\prime}\right)^{-1}\right]=\left(X_{q_{1}}^{-1}+X_{1 q}^{-1}\right)$. Since $q_{1}<_{1} q$, it follows from this equation and the equations last displayed that (36) is true.

For (37) our proof is rather lengthy and is composed of different parts. For convenience we shall present it under the following headings: $a$ first approach to the proof of (37); inequalities between products of elementary symmetric functions; proof of inequalities (46).

A first approach to the proof of (37). We treat the case ${ }_{1} q=r$ separately for a reason that is explained in the first footnote on page 895. When (33), with $\nu=k(>r)$, is applied to the set $X_{1} \ldots k$ under consideration, $X_{q_{1}} X_{1 q}<X_{q_{1}} X_{1 q}^{\prime}$ by (34). Therefore, with $q_{1}<{ }_{1} q=r$, it follows from (33) that

$$
\left(1 /\left(X_{1}^{\prime} X_{2}^{\prime} \cdots X_{r}^{\prime}\right)\right)<\left(1 /\left(X_{1} X_{2} \cdots X_{r}\right)\right)
$$

so that (37) holds for $p={ }_{1} q=r$. If $k=(r+1)$, our proof of (37) is complete. If $k>(r+1)$, we still need to prove that

(40) $\Sigma_{p, r}\left(1 / X^{\prime}\right)<\Sigma_{p, r}(1 / X)\left\{\begin{array}{l}\text { for } p={ }_{1} q, \cdots, k-1 \text { when }_{1} q>r>1 \\ \text { for } p=r+1, \cdots, k-1 \text { when }{ }_{1} q=r>1 .\end{array}\right.$

Since $q_{1}<{ }_{1} q \leqq p$, every set $X_{1} \ldots p$ under consideration is transformable. We shall prove (40) by establishing a set of inequalities that is formally different from, but equivalent to, (40). To obtain the set in question, namely (46) below, we first express $\Sigma_{p, r}(1 / \alpha), \alpha=X, X^{\prime}$, in (40) as a polynomial in $1 / \alpha_{q_{1}}$, $1 / \alpha_{1 q}$, and $1 /\left(\alpha_{q_{1}} \alpha_{1 q}\right)$, where by our hypotheses each of $q_{1},{ }_{1} q$ is a positive integer $\leqq p$. Let

$$
\Sigma_{\lambda}^{\prime}, \mu(1 / \alpha)\left\{\begin{array}{l}
\lambda=k-2 \text { or } p-2, \\
\mu=r, r-1, \text { or } r-2,
\end{array}\right.
$$

stand for the $\mu$ th elementary symmetric function of all of the reciprocals $1 / \alpha_{1}, 1 / \alpha_{2}, \cdots, 1 / \alpha_{\lambda+2}$, except $1 / \alpha_{q_{1}}$ and $1 / \alpha_{1} ;$; with the understanding that 


$$
\Sigma_{\lambda, \mu}^{\prime}(1 / \alpha)=\left\{\begin{array}{l}
0 \text { when } \lambda<\mu \text { and also when } \mu<0 \\
1 \text { when } \mu=0
\end{array}\right.
$$

Then

$$
\begin{aligned}
\Sigma_{p, r}(1 / \alpha) \equiv \Sigma_{p-2, r}^{\prime}(1 / \alpha) & +\left[\frac{1}{\alpha_{q_{1}}}+\frac{1}{\alpha_{1 q}}\right] \Sigma_{p-2, r-1}^{\prime}(1 / \alpha) \\
& +\frac{1}{\alpha_{q_{1}} \alpha_{1} q} \Sigma_{p-2, r-2}^{\prime}(1 / \alpha) .
\end{aligned}
$$

Further since (33) alters the values of only $X_{q_{1}}$ and $X_{1} q$, the following equalities are identities:

$$
\Sigma_{\lambda, \mu}^{\prime}\left(1 / X^{\prime}\right)=\Sigma_{\lambda, \mu}^{\prime}(1 / X) .
$$

By using (41) in (40) and then applying (42) in the resulting equation we now find that (40) is equivalent to

$$
\begin{aligned}
\left(\frac{1}{X_{q_{1}^{\prime}}^{\prime}}+\frac{1}{X_{1}^{\prime} q}\right) \Sigma_{p-2, r-1}^{\prime}(1 / X)+\frac{1}{X_{q_{1}^{\prime}}^{\prime} X_{1}^{\prime}{ }^{\prime}} \Sigma_{p-2, r-2}^{\prime}(1 / X) \\
<\left(\frac{1}{X_{q_{1}}}+\frac{1}{X_{1} q}\right) \Sigma_{p-2, r-1}^{\prime}(1 / X)+\frac{1}{X_{q_{1}} X_{1} q} \Sigma_{p-2, r-2}^{\prime}(1 / X) .
\end{aligned}
$$

A further inequality, equivalent to (43), will presently be obtained. Replacing both the left and right members of (38), a part of our present transformation (33), by their equivalents of the form (41) and using (42) in the resulting equation, we obtain an equation $\varepsilon$ that differs from (43) merely by having $=$ and $k$ in the place of $<$ and $p$, respectively, in (43). From (34) and $\varepsilon$ it follows that

$$
\frac{1}{X_{q_{1}^{\prime} X_{1} q}^{\prime}}=\frac{1}{X_{q_{1}} X_{1} q}-\epsilon, \frac{1}{X_{q_{1}^{\prime}}^{\prime}}+\frac{1}{X_{1} q}=\frac{1}{X_{q_{1}}}+\frac{1}{X_{1} q}+\Delta
$$

where $\epsilon$ and $\Delta$ are positive numbers. If we substitute in $\varepsilon$ for $\left(X_{q_{1}}^{\prime} X_{1 q}^{\prime}\right)^{-1}$ and $\left[\left(X_{q_{1}}^{\prime}\right)^{-1}+\left(X_{1 q}^{\prime}\right)^{-1}\right]$ their values of (44), simplify the resulting equation, and solve it for $\Delta$, we obtain

$$
\Delta=\frac{\epsilon \sum_{k-2, r-2}^{\prime}(1 / X)}{\Sigma_{k-2, r-1}^{\prime}(1 / X)},
$$

where $\Sigma_{k-2, r-1}^{\prime}(1 / X)>0$ since by hypothesis $k>r>1$ and $X_{i}>0$ (indeed $\left.X_{i} \geqq 1\right)$ for $i=1, \cdots, n$.

From (44) and (45) it now follows that (43), and therefore (40), is equivalent to

(46) $\Sigma_{k-2, r-1}^{\prime}(1 / X) \Sigma_{p-2, r-2}^{\prime}(1 / X)>\Sigma_{k-2, r-2}^{\prime}(1 / X) \Sigma_{p-2, r-1}^{\prime}(1 / X)(p$ as in $(40))$. 
If we can establish (46), it will follow from (46) and (39)* that (37) is true, and, since (38) was proved above, that Lemma 6 holds. We shall prove (46) immediately after we establish Lemma 8 below.

Inequalities between products of elementary symmetric functions. We use here a formula of Dresden for the product of two elementary symmetric functions, and for brevity in expressing symmetric functions we employ with Dresden the symbolic notation of partition theory. Thus the functions of $n$ variables $x_{1}, x_{2}, \cdots, x_{n}$ that are commonly denoted by $\Sigma\left(1 /\left(x_{1} x_{2} \cdots x_{s}\right)\right)$ and $\Sigma\left(1 /\left(x_{1}^{2} x_{2}^{2} \cdots x_{s}^{2}\right)\right)$ are here represented by $\left(1^{s}\right)$ and $\left(2^{s}\right)$, respectively. In this notation Dresden's formula is $\dagger$

$$
\left(1^{s_{1}}\right)\left(1^{s_{2}}\right)=\sum_{j=0}^{s_{2}}\left(\begin{array}{c}
s_{1}-s_{2}+2 j \\
j
\end{array}\right)\left(2^{s_{2}-j} 1^{s_{1}-s_{2}+2 j}\right) ; n \geqq s_{1} \geqq s_{2} \geqq 0 .+
$$

Remark. For future reference, it is to be noted here that with $x_{i} \geqq 1$, if $j=1$, or 2 , and if $s$ is an integer $\geqq 0$, then $\left(j^{s}\right)>0$ (cf. last footnote on this page) for the case $s=0$ ).

LEMMA 7. If $s_{1}$ and $s_{2}$ are integers such that $s_{1} \geqq s_{2} \geqq 0$, then

$$
\left(1^{s_{1}}\right)\left(1^{s_{2}}\right)>\left(1^{s_{1}+1}\right)\left(1^{s_{2}-1}\right) .
$$

From our definitions of $\Sigma_{i, j}(x), \S 12$, and $\left(1^{s}\right) \equiv \Sigma_{n, s}(1 / x)$, it follows that $\left(1^{0}\right)=1$ and $\left(1^{-1}\right)=0$. Hence Lemma 7 is true when $s_{2}=0$. Suppose that $s_{2} \geqq 1$. The product $\left(1^{s_{1}}\right)\left(1^{s_{2}}\right)$ is given by (47), from which it follows that

$$
\left(1^{s_{1}+1}\right)\left(1^{s_{2}-1}\right)=\sum_{j=0}^{s_{2}-1}\left(\begin{array}{c}
s_{1}-s_{2}+2+2 j \\
j
\end{array}\right)\left(2^{s_{2}-1-j} 1^{s_{1}-s_{2}+2+2 j}\right) .
$$

The exact numbers of terms that appear in the expansions of the right members of (47) and (48) are $\left(s_{2}+1\right)$ and $s_{2}$, respectively, and the type of term that is obtained by taking $j=\lambda$, where $0 \leqq \lambda \leqq\left(s_{2}-1\right)$, in (48) is gotten by setting $j=(\lambda+1)$ in (47). From the Remark just before Lemma 7 and the nature of the coefficients in the right members of (47) and (48), it is evident that no one of the $\left(2 s_{2}+1\right)$ terms just mentioned has a negative value. Now with $s_{1} \geqq s_{2} \geqq 1$ (which we are assuming), at least one term in the right

* We could have regarded the inequality in (46) as valid for the case $p={ }_{1} q=r$ (and thus included (39) in (46)); for with $k>r$ it follows from our definition of $\Sigma_{\lambda, \mu}^{\prime}(1 / X)$, just before (41), that if $p=r$ in (46) the resulting inequality is true. However, it seems desirable to have the proof above of (39). rather than allow this inequality to rest on our definition of $\Sigma_{\lambda, \mu}^{\prime}(1 / X)$.

$\dagger$ Cf. Arnold Dresden, On symmetric forms in $n$ variables, Annals of Mathematics, vol. 24 (1923), p. 227.

$\ddagger$ Since $n>r \geqq 1$ in this paper, we have no need for (47) in the following cases: (i) $n=s_{1}=s_{2}=0$; (ii) $n \geqq s_{1} \geqq 1$ and $s_{2}=0$. However, by defining $\left(\begin{array}{l}t \\ 0\end{array}\right)=1$ when $t$ is a non-negative integer, and using $\left(j^{0}\right)=1, j=1,2$, we find that in case (i), (4i) reduces to $1=1$; and in case (ii) to $\left(1^{1_{1}}\right)=\left(1^{1_{1}}\right)$. 
member of (48) is positive. Consequently, Lemma 7 follows from the inequality

$$
\left(\begin{array}{c}
s_{1}-s_{2}+2 \lambda+2 \\
\lambda+1
\end{array}\right)>\left(\begin{array}{c}
s_{1}-s_{2}+2 \lambda+2 \\
\lambda
\end{array}\right),
$$

whose validity is obvious from present hypotheses and known facts about the magnitudes of binomial coefficients.

LEMMA 8. If $u, v, \gamma$ are integers such that $u>v \geqq \gamma \geqq 1$, then

$$
\Sigma_{u, \gamma}(1 / x) \Sigma_{v, \gamma-1}(1 / x)>\Sigma_{u, \gamma-1}(1 / x) \Sigma_{v, \gamma}(1 / x) .
$$

When $\gamma=1, \Sigma_{t, \gamma-1}(1 / x)=1$ for $t=u$ or $v$; therefore the inequality to be proved is $\Sigma_{u, 1}(1 / x)>\Sigma_{v, 1}(1 / x)$. Since $u$ exceeds $v$ (and $x_{i} \geqq 1>0$ ), this relation is true. Now suppose $\gamma \geqq 2$. In the rest of this proof $\left(1_{1}^{s}\right)$ stands for the sth elementary symmetric function of the $v$ variables $1 / x_{i}(i=1,2, \cdots, v)$, and $E_{t}$ stands for the $t$ th such function of the $(u-v)$ variables $1 / x_{j}(j=v+1$, $v+2, \cdots, u)$. With this notation, we have the identities

$$
\begin{gathered}
\Sigma_{u, \gamma}(1 / x) \equiv\left(1_{1}^{\gamma}\right)+E_{1}\left(1_{1}^{\gamma-1}\right)+E_{2}\left(1_{1}^{\gamma-2}\right)+\cdots+E_{\gamma}\left(1_{1}^{0}\right), \\
\Sigma_{u, \gamma-1}(1 / x) \equiv\left(1_{1}^{\gamma-1}\right)+E_{1}\left(1_{1}^{\gamma-2}\right)+E_{2}\left(1_{1}^{\gamma-3}\right)+\cdots+E_{\gamma-1}\left(1_{1}^{0}\right),
\end{gathered}
$$

where $E_{i}=0$ when $i>(u-v)$. Consequently we only need to show that

$$
\begin{aligned}
& {\left[\left(1_{1}^{\gamma}\right)+E_{1}\left(1_{1}^{\gamma-1}\right)+E_{2}\left(1_{1}^{\gamma-2}\right)+\cdots+E_{\gamma}\left(1_{1}^{0}\right)\right]\left(1_{1}^{\gamma-1}\right)} \\
& >\left[\left(1_{1}^{\gamma-1}\right)+E_{1}\left(1_{1}^{\gamma-2}\right)+E_{2}\left(1_{1}^{\gamma-3}\right)+\cdots+E_{\gamma-1}\left(1_{1}^{0}\right)\right]\left(1_{1}^{\gamma}\right) .
\end{aligned}
$$

After subtracting $\left(1^{\gamma}\right)\left(1^{\gamma-1}\right)$ from both members of (49), we obtain the desired result by observing the following two facts: first, $E_{\gamma}\left(1_{1}^{0}\right)\left(1^{\gamma-1}\right)=$ $E_{\gamma}\left(1_{1}^{\gamma-1}\right) \geqq 0$; second, the coefficient of $E_{t}(t=1,2, \cdots, \gamma-1)$ in the left member of (49) exceeds, as we shall presently prove, the coefficient of $E_{t}$ in the right member. The second statement follows from the fact that when $\gamma \geqq 2$ and $1 \leqq t \leqq(\gamma-1), \quad\left(1_{1}^{\gamma-1}\right)\left(1_{1}^{\gamma-t}\right)>\left(1_{1}^{\gamma}\right)\left(1_{1}^{\gamma-t-1}\right) \quad$ (cf. Lemma 7 , with $\left.s_{1}=(\gamma-1), s_{2}=(\gamma-t)\right)$.

Proof of (46). Here by hypothesis $q_{1}<_{1} q$ and $r>1$. Hence inspection of the values which $p$ assumes in (46) shows that in these relations $(k-2)>(p-2) \geqq(r-1) \geqq 1$. Consequently, to prove that (46) is a special case of Lemma 8 , it suffices to define in Lemma 8 the $u, v, \gamma$ to be $(k-2),(p-2)$, $(r-1)$, respectively, and every $x_{i}(i=1, \cdots, u)$ to be equal to a different $X_{j}$ of (46), which contains exactly $(k-2)$ of the numbers $X_{1}, X_{2}, \cdots, X_{k}$ (cf. the definition of $\Sigma_{\lambda, \mu}^{\prime}(1 / X)$ between (40) and (41)).

A second step of the induction for (30) (or proof that $X_{1 \ldots k}, r<k \leqq(n-1)$, is not a set $\tau)$. Suppose the elements of $X_{1}^{\prime} \ldots \nu\left(\equiv X_{1}^{\prime} \ldots k\right.$ here $)$ are classified 
by writing $X^{\prime}, q^{\prime}, A^{\prime}, B^{\prime}$ in the place of $X, q, A, B$, respectively, in the classification of $\$ 15$, and that our transformation from the set $X_{1}^{\prime} \ldots k$ to a set $X_{1}^{\prime \prime} \ldots k$ is obtained by writing $X^{\prime}, X^{\prime \prime}, q^{\prime}, A^{\prime}, B^{\prime}$ in the place of $X, X^{\prime}, q, A, B$, respectively, in the definition of our transformation in $\$ 15$. Then $X_{1}^{\prime} \ldots k$ is of course transformable if, and only if, it contains at least one element of each of the classes $A^{\prime}, B^{\prime}$. We shall presently show (the proof beginning in the next paragraph) that in the case where $X_{1}^{\prime} \ldots k$ is not transformable, $X_{1}^{\prime} \ldots k$ is not a set $\tau$, so that $X_{1} \ldots k$ is not a set $\tau$ (cf. the Remark just after Lemma 6). In $\$ 18$ we shall prove that even if $X_{1}^{\prime} \ldots k$ is transformable, $X_{1}^{\prime} \ldots k$, and therefore $X_{1} \ldots k$, is not a set $\tau$.

By hypothesis $X_{1}^{\prime} \ldots k$ is a non-transformable set $\tau$; therefore it contains one or more elements of class $B^{\prime}$ and no element of class $A^{\prime}$ :

$$
X_{i}^{\prime} \leqq w_{i} \quad(i=1, \cdots, k),
$$

the sign $<$ holding here for at least one of the specified values of $i$. From these facts and the Definition of $\$ 14$, it follows that $X_{1}^{\prime} \ldots(k-1)$ is a set $\sigma$ that does not contain an element of class $A^{\prime}$, so that $X_{i}{ }^{\prime}=w_{i}(i=1, \cdots, k-1)$. These equalities together with (50) give

$$
X_{i}^{\prime}=w_{i} \quad(i=1, \cdots, k-1), \quad X_{k}^{\prime}<w_{k} .
$$

Consequently $X_{1}^{\prime} X_{2}^{\prime} \cdots X_{k}^{\prime}<w_{1} w_{2} \cdots w_{k}$. This inequality and relations (33), (34) give

$$
X_{1} X_{2} \cdots X_{k}<X_{1}^{\prime} X_{2}^{\prime} \cdots X_{k}^{\prime}<w_{1} w_{2} \cdots w_{k} .
$$

However, on the assumption that $X_{1}^{\prime} \ldots k$, with $r<k \leqq(n-1)$, is a " set $\tau$, it follows from (28) and (29) that

$$
\frac{b X_{1} X_{2} \cdots X_{k}-1}{a X_{1} X_{2} \cdots X_{k}} \geqq \Sigma_{k, r}(1 / X)>\Sigma_{k, r}(1 / w)=\frac{b w_{1} w_{2} \cdots w_{k}-1}{a w_{1} w_{2} \cdots w_{k}},
$$

so that $X_{1} X_{2} \cdots X_{k}>w_{1} w_{2} \cdots w_{k}$, which contradicts (51). Hence $X_{1}^{\prime} \ldots k$ is not a set $\tau$.

17. Further definitions and notation. In order to continue the induction of $\$ 16$, we extend our classification and transformation of elements and introduce further terminology.

Notation for successive sets of elements. Whatever be our transformation, if we apply it exactly once to a set $X_{1}^{(\alpha)} \ldots$, which we take to mean $X_{1} \ldots, X_{1}^{\prime} \ldots, X_{1}^{\prime \prime} \ldots, \cdots$ when $\alpha=0,1,2, \cdots$, respectively, the new set obtained will be called $X_{1}^{\alpha+1} \ldots$. 
General classification of elements. If a set $X_{1}^{(\alpha)} \ldots \nu$ is given, we obtain our classification of its elements (with our subscript notation) by writing $X^{(\alpha)}, q^{(\alpha)}, A^{(\alpha)}, B^{(\alpha)}$ in the place of $X, q, A, B$, respectively, in the classification of $\$ 15$; where of course $X^{(0)}, q^{(0)}, A^{(0)}, B^{(0)}$ stand for $X, q, A, B$, respectively.

General transformation. If $X_{1 \ldots \nu}^{(\alpha)}$ contains at least one element of each of the classes $A^{(\alpha)}, B^{(\alpha)}$, we define our transformation from $X_{1 \ldots \nu}^{(\alpha)}$ to $X_{1 \ldots \nu}^{(\alpha+1)}$ by merely writing $X^{(\alpha)}, X^{(\alpha+1)}, q^{(\alpha)}, A^{(\alpha)}, B^{(\alpha)}$ in the place of $X, X^{\prime}, q, A, B$, respectively, throughout the definition in $\$ 15$ of our transformation from $X_{1} \ldots$ to $X_{1}^{\prime} \ldots \nu$. For convenience in writing this general transformation, let $f, f^{\prime}, \theta$ stand for $X^{(\alpha)}, X^{(\alpha+1)}, q^{(\alpha)}$, respectively. Then our transformation from $f_{1} \ldots$, to $f_{1}^{\prime} \ldots$, is $t_{5}$ or $t_{6}$ :

$$
\begin{gathered}
\left(t_{5}\right) \quad f_{p}^{\prime}=f_{p}\left(p \neq \theta_{1},{ }_{1} \theta, p \leqq \nu\right), f_{\theta_{1}^{\prime}}^{\prime}=w_{\theta_{1}}^{\prime}, \Sigma_{\nu, r}\left(1 / f^{\prime}\right)=\Sigma_{\nu, r}^{\prime}(1 / f) ; \\
\left(t_{6}\right) \quad \text { “ } \quad, f_{1}^{\prime}=w_{: \nu}^{\prime},
\end{gathered}
$$

according as $t_{5}$ defines $f_{1 \theta}^{\prime}$ to be not greater than $w_{1 \theta}$ or greater than $w_{1} \theta$, respectively.

Remark. When (52) is applied the following relations hold:

$$
w_{\theta_{1}} \leqq f_{\theta_{1}^{\prime}}^{\prime}<f_{\theta_{1}}, \quad f_{1 \theta}<f_{1}^{\prime} \leqq w_{1} \theta .
$$

In words, one, and only one, element of class $A^{(\alpha)}\left[B^{(\alpha)}\right]$, namely the one with the smallest subscript, is always decreased [increased] by (52), and to a value not less [greater] than that of its corresponding element in the set $w_{1} \ldots$.

Intermediate and final sets of elements. Since $f_{1}^{\prime} \ldots \nu$ contains at least one more element of the set $w_{1} \ldots \nu$ than does $f_{1} \ldots \nu$ it follows that for any given set $X_{1} \ldots \nu$ there exists a smallest integer $\lambda \geqq 0$ such that $X_{1 \ldots \nu}^{(\lambda)}$ does not contain an element of each of the classes $A^{(\lambda)}, B^{(\lambda)}$ and is not transformable by (52). A set $X_{1 \ldots \nu}^{(\alpha)}, \alpha=1,2, \cdots, \lambda-1$, that is transformable will be called an intermediate set for $X_{1} \ldots \nu$, and $X_{1 \ldots \nu}^{(\lambda)}$ will be referred to as the final set for $X_{1} \ldots \nu$.

Exhaustive set of transformations. The set of $\lambda$ transformations which carries $X_{1} \ldots \nu$ into $X_{1 \ldots \nu}^{(\lambda)}$ will be called the exhaustive set for $X_{1} \ldots \nu$.

Remark. Since $X_{p} \geqq 1, p=1, \cdots, n$, and $w_{p} \geqq 1$, it is evident from the last Remark above that $X_{p}{ }^{(\alpha)} \geqq 1$ for $\alpha=0,1, \cdots, \lambda$. Thus it is true, as heretofore stated, that every element which we consider in our discussion of maximum numbers is at least as great as unity (cf. the last sentence before the Definition in \$14).

18. Continuation of the induction begun in $\$ 16$. In the case where $X_{1}^{\prime} \ldots k$ is not transformable we have shown in $\$ 16$ that $X_{1 \ldots k}$ is not a set $\tau$, and 
thus reached a contradiction. In making the demonstration of this section, we shall use a generalization of Lemma 6, namely Lemma 9 below, the proof of which we obtain by reasoning of the type that was used in establishing Lemma 6.

LEMMA 9. If $X_{1} \ldots k$ is a set $\tau$ or a transformable set $\sigma$ for which $r<k \leqq n$; if $f_{1} \ldots k$ stands either for $X_{1} \ldots k$ or for any one of its intermediate sets*; and if $\theta_{i},{ }_{j} \theta$ are related to $f_{1} \ldots{ }_{k}$ as $q_{i},{ }_{j} q$, respectively, are to $X_{1} \ldots{ }_{k} \dagger$ (cf. the classification of \$15), and if $t$ is a positive integer, application of (52) with $\nu=k$ to $f_{1} \ldots k$ yields a set $f_{1}^{\prime} \ldots{ }_{k}$ such that

$$
\begin{aligned}
& f_{\theta_{1}} f_{1} \theta<f_{\theta_{1}}^{\prime} f_{1}^{\prime}{ }^{\prime}, f_{\theta_{1}}{ }^{t}+f_{1^{\theta}}{ }^{t}<\left(f_{\theta_{1}}^{\prime}\right)^{t}+\left(f_{\theta^{\prime}}^{\prime}\right)^{t} ; \\
& \Sigma_{p, r}\left(1 / f^{\prime}\right) \leqq \Sigma_{p, r}(1 / w) \text { when } p=r, \cdots,{ }_{1} \theta-1 \text {; } \\
& \text { “ } \quad=\Sigma_{p, r}(1 / f) \text { when } r=1 \text { and } p={ }_{1} \theta, \cdots, k-1 \text {; } \\
& \text { “ } \quad<\Sigma_{p, r}(1 / f) \text { when } r>1 \text { and } p={ }_{1} \theta, \cdots, k-1 \text {; } \\
& \text { “ } \quad=\Sigma_{p, r}(1 / f) \text { when } p=k \text {. }
\end{aligned}
$$

Remark. Equality (57) holds because it is identical with the case $\nu=k$ of the last equation of both $t_{5}$ and $t_{6}$ in (52). If we establish relations (53) to (56) inclusive, it will be evident from them and the Definition of $\$ 14$ that if $f_{1} \ldots k$ is a set $\tau$ or a (transformable) set $\sigma$, then $f_{1}^{\prime} \ldots k$ is a set $\tau$ or a set $\sigma$, respectively.

Proof. The case $f=X, f^{\prime}=X^{\prime}, \theta=q$ of Lemma 9 has been established (in Lemma 6). Further if $X_{1}^{\prime} \ldots k$ is not transformable, it is the final set for $X_{1} \ldots k$, and Lemma 9 has exactly the content of Lemma 6. Hence the only case that we need to consider is where $X_{1}^{\prime} \ldots k$ is transformable. Suppose it is. If we can show (i) that $q_{1}^{\prime}<_{1} q^{\prime}$, the case $f=X^{\prime}, f^{\prime}=X^{\prime \prime}, \theta=q^{\prime}$ of (54) will obviously hold; if we can prove (ii) that

$$
X^{\prime}{ }_{q_{1}^{\prime}} \leqq X^{\prime}{ }_{1 q^{\prime}},
$$

the same case of relations (53) will follow from Lemma 1a; and then (55), (56) can be established by the method that was used in proving (36), (37), respectively. We prove (i) and (ii) presently.

(i). Since $X_{1}^{\prime} \ldots k$ is transformable and is either a set $\sigma$ or a set $\tau$ it follows from Lemma 5 that $q_{1}{ }^{\prime}<{ }_{1} q^{\prime}$.

* If $f_{1} \ldots k_{k}$ stands for $X_{1} \ldots k, f_{1} \ldots k_{k}$ is transformable (cf. Lemma 6). If $f_{1} \ldots k$ stands for any intermediate set for $X_{1} \ldots k$, it follows from the definition of intermediate set that $f_{1} \ldots k$ is transformable. Thus our hypothesis implies that $f_{1} \ldots k$ is transformable.

† If $f=X^{(\alpha)}$, then $\theta=q^{(\alpha)}$. Thus if $f_{1} \ldots k=X_{1 \cdots k}^{(\alpha)}$, then $f_{\theta_{1}}=X_{\delta_{1}}{ }^{(\alpha)}$ where $\delta_{1}=q_{1}{ }^{(\alpha)}$ and $f_{1} \theta=X_{1}{ }^{\delta(\alpha)}$ where ${ }_{1} \delta={ }_{1} q^{(\alpha)}$. 
(ii). To prove(58) it suffices to observe that, with $q_{1}^{\prime}<_{1} q^{\prime}, X_{q_{1}^{\prime}} \leqq X_{1 q^{\prime}}$ (the elements of $X$ being arranged in increasing order) and that

$$
X_{q_{1}^{\prime}}^{\prime \prime} \leqq X_{q_{1}^{\prime}}, X_{1 q^{\prime}} \leqq X_{1 q^{\prime}}^{\prime} \text { (cf. the Remark just below (52)). }
$$

Thus we find that the case $f=X^{\prime}, f^{\prime}=X^{\prime \prime}, \theta=q^{\prime}$ of Lemma 9 is true.

If $X_{1}^{\prime \prime} \ldots k$ is transformable, the method employed in the proof just completed can obviously be applied to show that the case $f=X^{\prime \prime}, f^{\prime}=X^{\prime \prime \prime}, \theta=q^{\prime \prime}$ of Lemma 9 is true; etc., until the final set $X_{1 \ldots k}^{(\lambda)}$ is obtained.

Completion of the proof that $X_{1} \ldots k$ is not a set $\tau$. If $X_{1 \ldots k}$ is a set $\tau$, so is the final set $X_{1}^{(\lambda)} \ldots k$ that we arrived at above (cf. the Remark just after Lemma 9). However, by argument of the type that was used in the last paragraph of $\$ 16$ one can show that $X_{1}^{(\lambda)} \ldots$ is not a set $\tau$.

Completion of the proof that (30) holds and that $X$ is a set $\sigma$. Recalling now that $X_{1} \ldots$ is a set $\sigma$ (cf. Lemma 4), we observe that since $X_{1} \ldots(r+1)$ is not a set $\tau, X_{1} \ldots(r+1)$ is a set $\sigma$; then, reasoning similarly, we find the sets $X_{1} \ldots(r+2), \cdots, X_{1 \cdots(n-1)}$ in this order (each set in its turn) to be sets $\sigma$. Hence (30) holds. Further, since $X \equiv X_{1} \ldots n$ is an $E$-solution, and $X_{1} \ldots(n-1)$ is a set $\sigma, X_{1} \ldots n$ is also a set $\sigma$, a fact which will be used in the proof of (32).

19. Proof of (31). What we wish to prove is that if $r=1$, then $\Sigma_{n-1,1}(1 / X)$ $\left\langle\Sigma_{n-1,1}(1 / w) .^{*}\right.$ We make the desired proof by treating the following two cases: (i) when $X_{1} \ldots(n-1)$ is not transformable; (ii) when $X_{1} \ldots(n-1)$ is transformable.

(i). Since $X$ and $w$ both satisfy (25), $X \neq w$ obviously implies that $X_{1} \ldots(n-1) \neq w_{1} \ldots(n-1)$. Now since $X\left(\equiv X_{1} \ldots n\right)$ is an $E$-solution in which $X_{1} \ldots(n-1)$ is not transformable and $\neq w_{1} \ldots(n-1)$, it follows from (30) that $X_{1} \ldots(n-1)$ contains one or more elements of class $A$ and no element of class $B$. Hence $\Sigma_{n-1,1}(1 / X)<\Sigma_{n-1,1}(1 / w)$.

(ii). Since $X_{1} \ldots(n-1)$ is transformable, $n \geqq 3$. From the hypotheses that $X$ is an $E$-solution and that $w$ is the Kellogg solution of the case $r=1$ of (25), it follows that if $X_{1} \neq w_{1}$, then $X_{1}>w_{1}$, so that $\Sigma_{1,1}(1 / X)<\Sigma_{1,1}(1 / w)$. With $m$ equal to a positive integer $\leqq(n-2)$, suppose that for every positive integral value of $p, 1 \leqq p \leqq m$, for which $X_{1 \ldots p} \neq w_{1} \ldots p, \Sigma_{p, 1}(1 / X)<\Sigma_{p, 1}(1 / w)$, and that $X_{1} \ldots m \neq w_{1} \ldots m$, so that

$$
\Sigma_{m, 1}(1 / X)<\Sigma_{m, 1}(1 / w) \equiv \frac{b w_{1} w_{2} \cdots w_{m}-1}{a w_{1} w_{2} \cdots w_{m}} \quad \text { (cf. (28)), }
$$

* One might think that if $r>1$ and if $X$ is an $E$-solution $\neq w$ of $(25)$ then $\Sigma_{n-1, r}(1 / X)<\Sigma_{n-1, r}(1 / w)$. That this is not always the case is shown in the following example.

Example. If $n=3, r=2, b=1$, and $c=9$, equation (25) becomes

$$
\frac{1}{x_{1} x_{2}}+\frac{1}{x_{3}}\left(\frac{1}{x_{1}}+\frac{1}{x_{2}}\right)=\frac{1}{9}
$$

and $w=(1,10,99)$. An $E$-solution $\neq w$ is $X=(2,5,63)$. Here $\Sigma_{2,2}(1 / X)=\Sigma_{2,2}(1 / w)$. 
and suppose that

$$
\Sigma_{m+1,1}(1 / X) \geqq \Sigma_{m+1,1}(1 / w) \equiv \frac{b w_{1} w_{2} \cdots w_{m+1}-1}{a w_{1} w_{2} \cdots w_{m+1}} .
$$

Then (59) and (60) imply that $X_{1} \ldots(m+1)$ is transformable. By (30) the sign $>$ does not hold in (60). Suppose $=$ holds there. Then $X_{1} \ldots(m+1)$ is a set $\sigma$ and when exhaustive applications of transformation (52) for $X_{1} \ldots(m+1)$ are made, the following relations hold (cf. (53)):

$$
X_{1} X_{2} \cdots X_{m+1}<X_{1}^{\prime} X_{2}^{\prime} \cdots X_{m+1}^{\prime} \leqq X_{1}^{(\lambda)} X_{2}^{(\lambda)} \cdots X_{m+1}^{(\lambda)}=w_{1} w_{2} \cdots w_{m+1},
$$

where $\lambda$ is the number of transformations in the exhaustive set for $X_{1} \ldots(m+1)$, and the equality sign holds between the last two products because of our hypothesis that the sign $=$ holds in (60). Thus

$$
X_{1} X_{2} \cdots X_{m+1}<w_{1} w_{2} \cdots w_{m+1} .
$$

One can now contradict (61) by observing that with (28), (29), and the case of equality in (60) holding, the following relations are true:

$$
\frac{b X_{1} X_{2} \cdots X_{m+1}-1}{a X_{1} X_{2} \cdots X_{m+1}} \geqq \Sigma_{m+1,1}(1 / X)=\frac{b w_{1} w_{2} \cdots w_{m+1}-1}{a w_{1} w_{2} \cdots w_{m+1}},
$$

so that $X_{1} X_{2} \cdots X_{m+1} \geqq w_{1} w_{2} \cdots w_{m+1}$ (contradiction). Hence (31) is true.

20. Proof of (32). Since $X$ is an $E$-solution $\neq w$, of (25), $X$ contains at least one element of each of the classes $A, B$. Consequently there exists at least one positive integer $p<n$ for which $X_{p} \neq w_{p}$. We shall complete the proof by considering the cases (i) and (ii) of $\$ 19$. The argument that was given under case (i) in the proof of (31) suffices in that case here. We treat case (ii) presently.

(ii). Here $X_{1} \ldots(n-1)$ is transformable and it has been shown to be a set $\sigma$ (cf. (30)). Hence $X\left[\equiv X_{1} \ldots n\right]$ is a transformable set $\sigma$, and (56) holds with $k=n$. Now let $g$ stand for $X$ itself or any one of its intermediate sets $X^{(\alpha)}$ for which $X_{n}{ }^{(\alpha)}=X_{n}$ except the last such set, and let it be denoted by $h$. Then it follows from the case $p=(k-1),=(n-1)$ here, of $(56)$, that

$$
\Sigma_{n-1, r}(1 / h)<\Sigma_{n-1, r}(1 / g)
$$

for every $g$, so that, in particular,

$$
\Sigma_{n-1, r}(1 / h)<\Sigma_{n-1, r}(1 / X) .
$$

Now since $X$ is a set $\sigma$, the following relations hold:

$$
\Sigma_{n-1, r}(1 / h)<\Sigma_{n-1, r}(1 / X) \leqq \Sigma_{n-1, r}(1 / w) .
$$


By hypothesis, $X_{n}=h_{n}$, while both $X$ and $h$ satisfy the equation

$$
\Sigma_{n-1, r}(1 / x)+\left(1 / x_{n}\right) \Sigma_{n-1, r-1}(1 / x)=b[(c+1) b-1]^{-1}(\mathrm{cf} .(25)) .
$$

From this equation and (62), then, $\Sigma_{n-1, r-1}(1 / h)>\Sigma_{n-1, r-1}(1 / X)$. Consequently to conclude that (32) holds we only need to prove that $\Sigma_{n-1, r-1}(1 / w)$ $\geqq \Sigma_{n-1, r-1}(1 / h)$. We shall establish the more descriptive relation

$$
\Sigma_{n-1, r-1}(1 / w)>\Sigma_{n-1, r-1}(1 / h) .
$$

From Lemma 9 and the definition of $h$ one observes that $h$ is a set $\sigma$ in which $h_{1} \ldots(n-1)$ is not transformable. Consequently, $h_{i} \geqq w_{i}(i=1, \cdots, n-1)$, and by (62) the sign $>$ holds in this relation for at least one of the specified values of $i$. Therefore (63) is true.

21. Summary of results obtained in Part 3. Since (30), (31), and (32) hold, Theorem 2, $\$ 12$, is true (cf. the first of two facts that are stated just before (30)). From Lemma 9 and the fact that every $E$-solution of (25) is a set $\sigma$ (cf. the last paragraph of $\$ 18$ ) it is evident that Lemma $3, \S 11$, holds with $u$ and (2) standing for $w$ and (25), respectively. Hence Theorem $3, \S 12$, is true.

\section{Part 4. Further possibilities of the Procedure of Part 3}

22. Why the procedure of Part 3 does not apply to equation (21). This will be shown by considering the following special case of (21):

$$
\Sigma_{3,1}(1 / x)+\Sigma_{3,3}(1 / x)=\frac{2}{7}\left[=\frac{b}{(c+1) b-1} \text { for } b=2 \text { and } c=3\right] \text {. }
$$

The Kellogg solution of $(64)$ is $w=(4,29,819)$ (cf. (23)).

Using notation that has been employed above, we observe that the present analog of transformation (33) is $t_{7}$ or $t_{8}$ :

$$
\begin{aligned}
\left(t_{7}\right) X_{p}^{\prime}=X_{p}\left(p \neq q_{1},{ }_{1} q, p \leqq \nu\right), X_{q_{1}^{\prime}} & =w_{q_{1}}, \\
\Sigma_{\nu, 1}\left(1 / X^{\prime}\right)+\Sigma_{\nu, 3}\left(1 / X^{\prime}\right) & =\Sigma_{\nu, 1}(1 / X)+\Sigma_{\nu, 3}(1 / X) ; \\
\left(t_{8}\right) \quad X_{p}^{\prime}=X_{p}\left(p \neq q_{1},{ }_{1} q, p \leqq \nu\right), X_{1}{ }^{\prime} & =w_{1}, \\
\Sigma_{\nu, 1}\left(1 / X^{\prime}\right)+\Sigma_{\nu, 3}(1 / X) & =\Sigma_{\nu, 1}(1 / X)+\Sigma_{\nu, 3}(1 / X),
\end{aligned}
$$

according as $t_{7}$ defines $X_{1}{ }^{\prime}$ to be not greater than $w_{1 q}$ or greater than $w_{1 q}$, respectively.

Since (64) is equivalent to

$$
\frac{1}{x_{1}}+\frac{1}{x_{2}}+\frac{1}{x_{3}}\left(1+\frac{1}{x_{1} x_{2}}\right)=\frac{2}{7},
$$

the analogs here of (30) and (32) are 


$$
\begin{gathered}
X_{1}^{-1}+X_{2}^{-1} \leqq w_{1}^{-1}+w_{2}^{-1}, \\
\left(X_{1} X_{2}\right)^{-1}<\left(w_{1} w_{2}\right)^{-1}, *
\end{gathered}
$$

respectively. By our theory for the case $r=1$ in Part 3, one can establish (66). The procedure of Part 3 does not enable one to prove (67), as we presently show.

Suppose that for equation (64) $X \neq w$ is an $E$-solution in which $q_{1}=1$ and ${ }_{1} q=2$ (such as $X=(5,12,427)$ ). If we apply $(65)$ to $X_{1} \ldots 3$, the product $X_{1} X_{2}$ will be increased (cf. Lemma 1a, §6), so that $\left(X_{1}^{\prime} X_{2}^{\prime}\right)^{-1}<\left(X_{1} X_{2}\right)^{-1}$, whereas the procedure of Part 3 would be to prove that a transformation on $X_{1} \ldots 2$ or $X_{1} \ldots 3$ increases the coefficient of $X_{3}^{-1}$ in the equation that results when $x$ in (64) is replaced by $X$. Thus the procedure of Part 3 would be to prove here that $\left[1+\left(X_{1}^{\prime} X_{2}^{\prime}\right)^{-1}\right]>\left[1+\left(X_{1} X_{2}\right)^{-1}\right]$, which would contradict the inequality just obtained by use of Lemma 1a.

For the theory of Part 3 we have found no modification that will yield for (21) results analogous to those which we have established for (25). Nevertheless, we have not exhibited an example in which we are able to prove that such results do not hold. In the next section we state the additional information that we have about (21) and the theorems which our procedure yields relative to another equation very much like (21).

23. Statement (without proof) of further results obtainable by our method. We include in our statements here the results that are expressed in Theorem $i(i=2,3)$.

THEOREM 4. If in (21) either $\lambda_{p}=1(p=r+1, \cdots, s)$, or $\lambda_{r+1}$ is an integer $\geqq 0$ and $\lambda_{p}=0(p=r+2, \cdots, s)$, the largest number that exists in any $E$-solution of the resulting equation (21) is the $w_{n}$ of the corresponding solution $w$ defined in (23). Furthermore, in each of these cases $w_{n}$ appears in but one E-solution of the equation (21) in question.

Theorem 5. In each of the two cases of Theorem 4 , if $X$ is an E-solution of equation (21) and is different from the $w$ of that equation, then $P(X)<P(w)$ (cf. the definition of $P(x)$ in the third paragraph of \$2).

Remark. When $r=1, s=n$, and $\lambda_{p}=1(p=2, \cdots, n)$ equation (21) is equivalent to

$$
\prod_{i=1}^{n}\left(1+1 / x_{i}\right)=1+b / a, \quad a \equiv[(c+1) b-1]
$$

* If in (64) $x$ is replaced by $X$, the coeflicient of $X_{3}^{-1}$ is $\left[1+\left(X_{1} X_{2}\right)^{-1}\right]$. Thus it is apparent that $(67)$ is a simplified form of the inequality

$$
1+\left(X_{1} X_{2}\right)^{-1}<1+\left(w_{1} w_{2}\right)^{-1}
$$


and if we employ the notation $\pi_{i}(\alpha) \equiv\left(1+\alpha_{1}\right)\left(1+\alpha_{2}\right) \cdots\left(1+\alpha_{i}\right)$, solution (23) assumes the elegant form

$$
w_{1}=(c+1), w_{i+1}=a \pi_{i}(w)+1(i=1, \cdots, n-2), w_{n}=a \pi_{n-1}(w) .
$$

If further $b=c=1$, solution $w$ is given by

$$
w_{i}=2^{2 i-1}(i=1, \cdots, n-1), w_{n}=2^{2^{n-1}}-1 .
$$

Thus Theorem 4 and Theorem 5 give interesting results about equation (68).

Results relative to another elementary symmetric equation much like (21). Consider the equation

$$
\lambda_{r} \Sigma_{n, r}(1 / x)+\lambda_{r+1} \Sigma_{n, r+1}(1 / x)+\cdots+\lambda_{s} \Sigma_{n, s}(1 / x)=1,
$$

in which $r, s, n$, and $\lambda_{r}$ are positive integers with $r<s \leqq n$, and $\lambda_{p}(p=r+1$, $\ldots, s)$ is an integer $\geqq 0$. That neither of equations (69) and (21) includes the other is made clear by the following two statements, each of which is obviously true: first, (69) and not (21) contains the equation $3 \Sigma_{3,3}(1 / x)+$ $5 \Sigma_{3,2}(1 / x)=1$; second, (21) and not (69) contains the equation $\Sigma_{3,3}(1 / x)+$ $5 \Sigma_{3,2}(1 / x)=2 / 5$.

For equation (69) it turns out that the Kellogg solution is one in positive integers, namely $x=w$, where

$$
\begin{aligned}
& w_{p}=1 \quad(p=1, \cdots, r-1), \quad w_{r}=\lambda_{r}+1, \\
& w_{p+1}=\lambda_{r} \Sigma_{p, p-r+1}(w)+\lambda_{r+1} \Sigma_{p, p-r}(w)+\cdots+\lambda_{s} \Sigma_{n-1, n-s+1}(w)+1 \quad(p=r, \cdots, n-2), \\
& \\
& w_{n}=\lambda_{r} \Sigma_{n-1, n-r}(w)+\lambda_{r+1} \Sigma_{n-1, n-r+1}(w)+\cdots+\lambda_{s} \Sigma_{n-1, n-s}(w) .
\end{aligned}
$$

By the methods of Part 3, we have proved that if in Theorem $i(i=4,5)$, (21) and (23) are replaced by (69) and (70), respectively, the resulting statements are true.

\section{Part 5. Applications}

24. On the convergence of a type of series. From the fact that every $E$ solution $X$ of (25) is a set $\sigma$ (cf. (30)), it follows that among all infinite series with $p$ th term equal to $\left(1 / x_{r+p-1}\right) \Sigma_{r+p-2, r-1}(1 / x)$, where the $x$ 's are positive integers such that

$$
\Sigma_{u, r}(1 / x)<(b / a), \quad a \equiv[(c+1) b-1], \quad u=r, r+1, \cdots,
$$

$b$ and $c$ being any positive integers, there is no series which converges to (b/a) more rapidly than does the one that is obtained by letting $n$ increase indefinitely* in (25) and then taking $x_{p}$ equal to $w_{p}(p=1, \cdots, n-1)$ of

* Kellogg has mentioned applications of Kellogg solutions (not so named by him) to series and to mapping (loc. cit. in third footnote on p. 876). 
(26). When $r=1$ the series thus obtained converges to $(b / a)$ more rapidly (cf. (31)) than does any other series of the specified type.

25. An answer to a question of Curtiss concerning a maximum number. A corollary of Theorem 3 defines unique maximum values for the coefficients $c_{i}$ of rational, integral, algebraic equations of the $n$th degree, of the form

$$
x^{n}-c_{1} x^{n-1}+c_{2} x^{n-2}-+\cdots+(-1)^{n} c_{n}=0,
$$

whose $n$ roots constitute an $E$-solution of (25). This answers a question which was raised by Curtiss*; in fact, it does more since his inquiry was about positive integral solutions rather than $E$-solutions.

26. Maximum numbers and minimum numbers associated with a problem in physics. Our results contain a considerable amount of information about the following problem in physics. If the resistance in the $i$ th wire of a set of $n$ wires which are connected in parallel in an electric circuit is $x_{i}$, the total resistance $x$ in the circuit is, as is well known, $\dagger$ given by the equation $x^{-1}=$ $\Sigma_{n, 1}(1 / x)$. For a given positive integral value of $x$, Theorem $2, \S 12$, gives the maximum value that any one of the $x_{i}$ can assume in any $E$-solution of this equation; Theorem 3, §12, the maximum value of $\Sigma_{n, r}(x)$ in any $E$-solution; Theorem $1, \S 2$, the least value that $\Sigma_{n, r}(x)$ can have in any positive solution. In any $E$-solution of the given equation the minimum value of any $x_{i}$ when $n>1$ is obviously $(x+1)$ and the smallest value of the largest $x_{i}$ is $n x$.

27. An upper bound for a perfect number with exactly $n$ divisors less than itself. It has been pointed out $\ddagger$ that a perfect number with exactly $n$ divisors less than itself, unity included, can not exceed the value which $w_{n}$ assumes in (26) in the case $r=a=b=1$. Suppose that $\alpha_{n}$ is a perfect number which has $n$ numbers $1, \alpha_{1}, \alpha_{2}, \cdots, \alpha_{n-1}$ as divisors, and no other divisor except $\alpha_{n}$ itself. Then from the definition of perfect number it follows that $\alpha_{n}=\left[1+\Sigma_{n, 1}(\alpha)\right] / 2$. Since $\Sigma_{n, 1}(w)$ is an upper bound for $\Sigma_{n, 1}(\alpha)$ (cf. Theorem 3 ), we conclude that a perfect number with exactly $n$ divisors less than itself can not exceed $B \equiv\left[1+\Sigma_{n, 1}(w)\right] / 2$. If we can show that $B<w_{n}$ for all positive integral values of $n>2$, we shall have in $B$ a better upper bound than $w_{n}$. This we prove presently.

In our special case $(r=a=b=1)$ of $(26), w_{n}=w_{1} w_{2} \cdots w_{n-1}$, which equals $w_{n-1}\left(w_{n-1}-1\right)$, as follows from (26). We desire to establish the inequality $w_{n}>B$ or its equivalent $w_{n-1}\left(w_{n-1}-1\right)>\left(w_{1}+w_{2}+\cdots+w_{n-1}+1\right)$. This relation is obviously true if $w_{n-1}\left(w_{n-1}-1\right)>\left[(n-1) w_{n-1}+1\right]$; or, indeed,

* Cf. D. R. Curtiss, p. 864 of the paper cited in first footnote on p. 883. 1917).

† Cf., for example, Arthur L. Kimball's $A$ College Text in Physics, p. 428 (2d edition revised,

$\ddagger$ Cf. the article of Curtiss referred to in third footnote on p. 876 . 
if $w_{n-1}>n$. Now from the case $r=a=b=1$ of (26),

$$
w_{n-1} \equiv\left(w_{1} w_{2} \cdots w_{n-2}+1\right) \geqq\left(2^{n-2}+1\right) ;
$$

and one can easily prove that $\left(2^{n-2}+1\right)>n$ if $n>3$. In the case $n=3, w_{3}=$ $2^{-1}\left(w_{1}+w_{2}+w_{3}+1\right)=6$. Hence $w_{n}>B$ when, and only when, $n>3$ (no value less than 3 being admitted for $n$ ).

We shall now give a descriptive comparison of the upper bounds $B$ and $w_{n}$. For $n=5, R_{n} \equiv\left(B / w_{n}\right)<0.52$. We prove below that $R_{n}$ decreases as $n(>2)$ increases through positive integral values, and that the limit of $R_{n}$ as $n$ increases indefinitely through such values is $2^{-1}$; from these facts it will be clear that for $n>4, B$ is (only) slightly greater than $2^{-1} w_{n}$.

Proof that $R_{n}$ decreases as $n>2$ increases. With $m$ equal to an integer $>2$, the inequality $R_{m}>R_{m+1}$ is, by (26), equivalent to

$$
\begin{gathered}
\frac{1+w_{1}+w_{2}+\cdots+w_{m-1}+\left(w_{n}-1\right)}{w_{m}-1} \\
>\frac{1+w_{1}+w_{2}+\cdots+w_{m}+w_{m+1}}{w_{m+1}} .
\end{gathered}
$$

Using the fact that if $n=(m+1)$ then $w_{m+1}=w_{m}\left(w_{m}-1\right)$, we find that $(71)$ is equivalent to

$$
\frac{1+w_{1}+w_{2}+\cdots+w_{m-1}}{w_{m}-1}>\frac{1+w_{1}+w_{2}+\cdots+w_{m}}{w_{m}\left(w_{m}-1\right)},
$$

and, therefore, to $\left(w_{m}-1\right)\left(1+w_{1}+w_{2}+\cdots+w_{m-1}\right)>w_{m}$, which obviously holds since $\left(1+w_{1}+w_{2}+\cdots+w_{m-1}\right)$ and $w_{m}$ both exceed 2 .

Proof that the limit of $R_{n}$ is $2^{-1}$. From the definition of $B$,

$$
R_{n}=\frac{1+w_{1}+w_{2}+\cdots+w_{n-2}}{2 w_{n}}+\frac{w_{n-1}}{2 w_{n}}+\frac{1}{2} .
$$

The middle fraction in the right member of (72) reduces by the equality $w_{n}=w_{n-1}\left(w_{n-1}-1\right)$ to an expression whose limit as $n$ approaches infinity is zero (cf. (26)). In finding the limit as $n$ increases indefinitely of the first fraction in that right member, we apply the known fact that

$$
\left(1+w_{1}+w_{2}+\cdots+w_{n-2}\right) \leqq\left[(n-2)+w_{1} w_{2} \cdot w_{n-2}\right] . *
$$

* Cf. American Mathematical Monthly, March, 1930, Theorem 1a, p. 137. 
From this relation it is now evident that the desired proof will be made if we show that

$$
\lim _{n \rightarrow \infty} \frac{n-2}{w_{n-1}\left(w_{n-1}-1\right)}=0 ; \quad \lim _{n \rightarrow \infty} \frac{w_{1} w_{2} \cdots w_{n-2}}{w_{n}}=\lim _{n \rightarrow \infty} \frac{1}{w_{n-1}}=0 .
$$

Since $w_{n-1}>n$ (cf. the second paragraph of this section), these limits are zero.

NORTHWESTERN UNIVERSTTY, Evanston, Ill. 